\title{
Läuterung der Verwaltungsrechtstheorie
}

\author{
- Aufgaben und Methoden der Wissenschaft vom Recht der Verwaltung - \\ Stefan Haack*
}

A. Prozesse der Selbstfindung in der Verwaltungsrechtswissenschaft

B. Die Unterscheidung von Rechtmäßigkeit und Rechtswidrigkeit als Erkenntnisziel der Verwaltungsrechtswissenschaft

I. Die Leistung der modernen Rechtswissenschaft, das öffentliche Recht von den übrigen Maßstäben des hoheitlichen Handelns zu unterscheiden....

II. Schwierigkeiten und Schlussfolgerungen, die sich aus einer solchen Bestimmung des rechtswissenschaftlichen Erkenntnisinteresses ergeben

C. Vier Schritte zu einer Läuterung des verwaltungsrechtstheoretischen Denkens....

I. Die Beschränkung einer rechtspolitischen Inanspruchnahme der juristischen Arbeit....
II. Die Beschränkung eines Imports sozialwissenschaftlicher Erkenntnisse und Theorien in das verwaltungsjuristische Denken...

III. Die Beschränkung einer auf die Praxis der Verwaltung fixierten Herangehensweise....

IV. Die Beschränkung von Reflexionen über die historische Bedingtheit des Rechts

D. Die Situation des juristischen Konflikts und der Klage als Dreh- und Angelpunkt der Verwaltungsrechtstheorie. . 440

E. Schlussbemerkungen: Gewissheiten in der Rechtswissenschaft durch die Konzentration auf den Prozess als den Kern des juristischen Denkens.

\section{A. Prozesse der Selbstfindung in der Verwaltungsrechtswissenschaft}

Eine Vielzahl von neuen Büchern zu den Aufgaben und zu den Methoden der Wissenschaft vom öffentlichen Recht ${ }^{1}$ illustriert, dass diese zur Zeit in einer Phase der Selbstfindung steckt. Was in der Wissenschaft vom Verfassungsrecht seit jeher mehr oder weniger intensiv diskutiert worden ist, hat nun auch die Verwaltungsrechtstheorie eingeholt: ${ }^{2}$ man sucht sich zu verständigen und $\mathrm{zu}$ vergewissern über das Erkenntnisziel und die Erkenntnismethoden ${ }^{3}$ - und fürchtet dabei wohl (obgleich man dies nur selten ausspricht), dass die Lehre vom Verwaltungsrecht als unwissen-

* Prof. Dr. Stefan Haack lehrt Öffentliches Recht an der Universität Bonn.

1 Genannt seien insbesondere die Sammelbände von Engel/Schön (Hrsg.), Das Proprium der Rechtswissenschaft, Tübingen 2007; Funke/Lüdemann (Hrsg.), Offentliches Recht und Wissenschaftstheorie, Tübingen 2009; Jestaedt/Lepsius (Hrsg.), Rechtswissenschaftstheorie, Tübingen 2008; Kirchhof/Magen/ Schneider (Hrsg.), Was weiß Dogmatik?, Tübingen 2012 sowie Schmidt-Aßmann/Hoffmann-Riem (Hrsg.), Methoden der Verwaltungsrechtswissenschaft, Baden-Baden 2004.

2 C. Möllers, Methoden, in: Hoffmann-Riem/Schmidt-Aßmann/Voßkuhle (Hrsg.), Grundlagen des Verwaltungsrechts, Bd. I, 2. Aufl., München 2012, \$3 Rn. 21; A. Voßkuble, Methode und Pragmatik im Öffentlichen Recht, Vorüberlegungen zu einem differenziert-integrativen Methodenverständnis am Beispiel des Umweltrechts, in: FS R. Schmidt, Tübingen 2006, S. 171 (176).

3 Kritisch hierzu U. Vosgerau, Rechtswissenschaft als Theorie der Theorie oder als praktische Kulturwissenschaft, in: Funke/Lüdemann (Hrsg.), Öffentliches Recht und Wissenschaftstheorie, Tübingen 2009, S. 201 (208 ff.). 
schaftlich entlarvt werden könnte. ${ }^{4}$ Über die Gründe für diese Entwicklung wurde bereits viel Kluges geschrieben, ${ }^{5}$ das hier nicht wiederholt werden soll. Hinzugefügt sei nur, dass man das Fehlen einer Theorie der Rechtswissenschaft im öffentlichen Recht möglicherweise auch deshalb stärker als im Zivilrecht oder im Strafrecht empfindet, ${ }^{6}$ weil die Wissenschaft vom öffentlichen Recht mit den Fragen des Rechtsbegriffes und der rechtlichen Geltung häufiger und unmittelbarer als die anderen Teildisziplinen befasst ist - aus dem Bereich des Verwaltungsrechts seien hierzu solch unterschiedliche Themenkreise erwähnt wie die Rechtsnatur der Verwaltungsvorschriften, das Fortbestehen von Beamtenverhältnissen und der hieraus sich ergebenden Ansprüche nach dem politischen Umbruch oder die Umsetzung von internationalen und transnationalen Regelungswerken (zum Umweltschutz, zum Luftverkehr oder zum Kapitalmarkt) im innerstaatlichen Recht. Ein juristisches Denken, das sich auf die hergebrachten Methoden der Rechtsauslegung und der Rechtsanwendung beschränkt, gelangt hierbei möglicherweise an seine Grenzen - ohne dass hierdurch die Berechtigung und die Unersetzlichkeit der traditionellen dogmatischen Arbeit in Frage gestellt werden könnten.

Gänzlich verschieden erscheinen indes die Strategien und die Mittel, die in der Debatte nahegelegt worden sind, um die Wissenschaft vom Verwaltungsrecht für die bevorstehenden Herausforderungen zu wappnen: während manche Autoren Ver-

4 Von einem „Trauma“ spricht insofern (bezogen auf die bekannten Stellungnahmen J. von Kirchmanns und R. von Jherings zum wissenschaftlichen Stellenwert der Jurisprudenz) M. Jestaedt, Wissenschaftliches Recht, Rechtsdogmatik als gemeinsames Kommunikationsformat von Rechtswissenschaft und Rechtspraxis, in: Kirchhof/Magen/Schneider (Hrsg.), Was weiß Dogmatik?, Tübingen 2012, S. 117 (122 ff.); vgl. auch dens., „Öffentliches Recht“ als wissenschaftliche Disziplin, in: Engel/Schön (Hrsg.), Das Proprium der Rechtswissenschaft, Tübingen 2007, S. 241 (242); dens., Braucht die Wissenschaft vom Öffentlichen Recht eine fachspezifische Wissenschaftstheorie?, in: Funke/Lüdemann (Hrsg.), Öffentliches Recht und Wissenschaftstheorie, Tübingen 2009, S. 17 (20); I. Appel, Das Verwaltungsrecht zwischen klassischem dogmatischen Verständnis und steuerungswissenschaftlichem Anspruch , VVDStRL 67 (2008), S. 226 (234); A. von Arnauld, Die Wissenschaft vom Öffentlichen Recht nach einer Öffnung für sozialwissenschaftliche Theorie, in: Funke/Lüdemann (Hrsg.), Öffentliches Recht und Wissenschaftstheorie, Tübingen 2009, S. 65 (85); O. Lepsius, Kritik der Dogmatik, in: Kirchhof/Magen/ Schneider (Hrsg.), Was weiß Dogmatik?, Tübingen 2012, S. 39 (60 f.) und C. Möllers, Vorüberlegungen zu einer Wissenschaftstheorie des öffentlichen Rechts, in: Jestaedt/Lepsius (Hrsg.), Rechtswissenschaftstheorie, Tübingen 2008, S. 151 (164 ff.).

5 Vgl. Jestaedt, Wissenschaftstheorie (Fn. 4), S. 41 f. und C. Möllers, Braucht das öffentliche Recht einen neuen Methoden- und Richtungsstreit?, VerwArch 90 (1999), S. 187.

6 So Jestaedt, Wissenschaftstheorie (Fn. 4), S. 41; vgl. dazu ferner C. Bumke, Die Entwicklung der verwaltungsrechtswissenschaftlichen Methodik in der Bundesrepublik Deutschland, in: Schmidt-Aßmann/ Hoffmann-Riem (Hrsg.), Methoden der Verwaltungsrechtswissenschaft, Baden-Baden 2004, S. 73 (78f.) sowie (aus österreichischer Sicht) G. Winkler, Die Wissenschaft vom Verwaltungsrecht, in: Ermacora/Winkler/Koja/Rill/Funk (Hrsg.), Allgemeines Verwaltungsrecht, Wien 1979, S. 3 (23). 
waltungsrechtswissenschaft als Steuerungswissenschaft ${ }^{7}$ oder als Governance-Forschung ${ }^{8}$ betreiben, plädieren andere für eine erkenntnistheoretische Selbstdisziplinierung des Fachs ${ }^{9}$ und fordern wiederum andere eine vorsichtige Öffnung und Modernisierung der traditionellen verwaltungsrechtlichen Lehre. ${ }^{10}$ Es gibt indes einen Weg, dessen Erfolgsaussicht bislang noch nicht hinreichend klar erkannt worden ist: gemeint ist damit die radikale Reduktion des verwaltungsrechtlichen Denkens auf seinen harten juristischen Kern; ${ }^{11}$ gemeint ist mit anderen Worten eine Läuterung der Verwaltungsrechtstheorie anhand allgemeiner Prämissen und Eigenheiten der rechtswissenschaftlichen Arbeit. Dass diese Formulierung weder als überhöht noch als respektlos gegenüber den Leistungen der anderen Strömungen in der Verwaltungsrechtswissenschaft eingestuft werden muss, mögen die folgenden Abschnitte zeigen. Dreh- und Angelpunkt einer solchen Läuterung muss das besondere Erkenntnisziel der Wissenschaft vom Verwaltungsrecht sein: es lassen sich von hier aus so-

7 So u.a. M. Eifert, Zum Verhältnis von Dogmatik und pluralisierter Rechtswissenschaft, in: Kirchhof/ Magen/Schneider (Hrsg.), Was weiß Dogmatik?, Tübingen 2012, S. 79 (92 ff.); E. Schmidt-Aßmann, Das allgemeine Verwaltungsrecht als Ordnungsidee, 2. Aufl., Berlin (u.a.) 2006, S. 18; A. Voßkuble, Neue Verwaltungsrechtswissenschaft, in: Hoffmann-Riem/Schmidt-Aßmann/Voßkuhle (Hrsg.), Grundlagen des Verwaltungsrechts, Bd. I, 2. Aufl., München 2012, $\mathbb{1}$ Rn. 16 ff.; ders., Pragmatik (Fn. 2), S. 180 f.; vgl. dazu ausführlich Appel, Steuerungswissenschaft (Fn. 4), S. 226 ff.; M. Eifert, Das Verwaltungsrecht zwischen klassischem dogmatischen Verständnis und steuerungswissenschaftlichem Anspruch, VVDStRL 67 (2008), S. 286 ff.; S. Meyer, Fordert der Zweck im Recht wirklich eine „Neue Verwaltungsrechtswissenschaft"?, Zugleich ein Vorschlag zur Dogmatik des Verwaltungsermessens, VerwArch 101 (2010), S. 351; R. Schröder, Verwaltungsrechtsdogmatik im Wandel, Tübingen 2007, S. 192 ff.; I. Spiecker genannt Döhmann, Das Verwaltungsrecht zwischen klassischem dogmatischem Verständnis und steuerungswissenschaftlichem Anspruch, DVBl. 2007, S. 1074 sowie H. Treiber, Verwaltungsrechtswissenschaft als Steuerungswissenschaft - eine „Revolution auf dem Papier“?, Anmerkungen zu einem intendierten Paradigmawechsel und zur „Kühnheit“ von Schlüsselbegriffen (Teil 1), KJ 41 (2007), S. 328 ff. und (Teil 2), KJ 42 (2008), S. 48 ff.

8 So u.a. G. F. Schuppert, Verwaltungsrecht und Verwaltungswissenschaft im Wandel. Von Planung über Steuerung zu Governance?, AöR 133 (2008), S. 79 (100 ff.); C. Franzius, Governance und Regelungsstrukturen, VerwArch 97 (2006), S. 186; H.-H. Trute/D. Kühlers/A. Pilniok, Governance als verwaltungsrechtswissenschaftliches Analysekonzept, in: Schuppert/Zürn (Hrsg.), Governance in einer sich wandelnden Welt, Wiesbaden 2008, S. 173 und H.-H. Trute/W. Denkhaus/D. Kühlers, Governance in der Verwaltungsrechtswissenschaft, Die Verwaltung 37 (2004), S. $451 \mathrm{ff}$.

9 Siehe dafür insbesondere Jestaedt, Disziplin (Fn. 4), S. 267 ff.; ders., Wissenschaftstheorie (Fn. 4), S. $33 \mathrm{ff}$.

10 In diese Richtung von Arnauld, Wissenschaft (Fn. 4), S. 99 ff. (bezogen auf eine Öffnung der Wissenschaft vom Verwaltungsrecht gegenüber den Sozialwissenschaften, siehe dazu unten: C.II.); ferner auch W. Kahl, Über einige Pfade und Tendenzen in Verwaltungsrecht und Verwaltungsrechtswissenschaft - Ein Zwischenbericht, Die Verwaltung 42 (2009), S. 463 (498) (unter stärkerer Einbeziehung einer „steuerungswissenschaftlichen Handlungsperspektive“ - der Unterschied zu den steuerungswissenschaftlichen Ansätzen der Neuen Verwaltungsrechtswissenschaft ist hier und bei anderen Autoren lediglich ein gradueller).

11 Versuche, der Verwaltungsrechtstheorie ein kohärentes theoretisches Fundament zugrunde zu legen, das aus den Prämissen des juristischen Denkens heraus konzipiert worden ist, hat es freilich gegeben; auf die Lehre vom Rechtsverhältnis, die insbesondere in ihrer Formulierung durch N. Achterberg als ein solcher Vorstoß eingestuft werden kann, kommen wir an späterer Stelle zurück (siehe unten: D.). Einen weiteren interessanten Versuch, der allerdings kaum rezipiert worden ist, stellt die „Prinzipientheorie des Verwaltungsrechts" von J. H. Park (Rechtsfindung im Verwaltungsrecht, Berlin 1999, S. 287 ff.) dar. 
wohl die Frage nach ihren Aufgabenfeldern als auch die Besonderheiten ihres methodischen Rüstzeugs erschließen.

\section{B. Die Unterscheidung von Rechtmäßigkeit und Rechtswidrigkeit als Erkenntnisziel der Verwaltungsrechtswissenschaft}

\section{Die Leistung der modernen Rechtswissenschaft, das öffentliche Recht von den übrigen Maßstäben des hoheitlichen Handelns zu unterscheiden}

Jede rechtswissenschaftliche Beschäftigung mit dem Verwaltungsrecht muss sich, solange sie als eine solche anzusehen sein soll, eine zentrale Frage vorlegen - es ist dies die juristische Frage schlechthin, was als rechtmäßig gilt. ${ }^{12}$ Die Aufgabe der Verwaltungsrechtswissenschaft besteht darin zu untersuchen, wie man zu einer Antwort auf diese Frage gelangt; ${ }^{13}$ gerade dies ist ihr originäres Erkenntnisinteresse. ${ }^{14}$ Ungeachtet des beträchtlichen Klärungsbedarfs, der sich an diese Aussage knüpft, ist zu bemerken, dass es sich bei der Jurisprudenz um die einzige Wissenschaft handelt, die sich diese Aufgabe vorlegt ${ }^{15}$ - und mehr noch: will man die Wissenschaftszweige von ihrem Erkenntnisziel her unterscheiden (was aus wissenschaftstheoretischer Sicht unerlässlich erscheint), so stellt die Anleitung zur Unterscheidung von Rechtmäßigkeit und Rechtswidrigkeit per definitionem die einzige Aufgabe dieser Wissenschaft dar. Mit anderen Worten: sobald man bereit ist anzuerkennen, dass für den Bereich der Verwaltung geltendes Recht existiert (was richtigerweise wohl niemand bestreitet), muss man einen speziellen, allein auf das juristische Denken fixierten Wissenschaftszweig als berufen ansehen, dem Juristen deutlich zu machen, wie man auf dem Terrain des behördlichen Handelns Rechtmäßigkeit und Rechtswidrigkeit identifi-

12 Um dies für richtig zu halten, muss man kein Positivist sein. Eine Bestimmung des Erkenntnisziels der Rechtswissenschaft, wie sie hier vorgeschlagen wird, erscheint zunächst einmal überhaupt nicht an eine bestimmte Vorstellung davon geknüpft, wie das Recht beschaffen sein sollte. Insofern ist nichts dagegen einzuwenden, wenn man in der Literatur die Entwicklung einer zeitgemäßen Rechtsvorstellung als „eine Sache der rechtstheoretischen Innovationsforschung“ bezeichnet und dies als eine Aufgabe der Rechtswissenschaft ansieht (M. Pöcker, Fehlende Kommunikation und die Folgen, Das heutige Verhältnis dogmatischer Rechtswissenschaft zu rechtstheoretischer Innovationsforschung, Die Verwaltung 37 [2004], S. 509 [510]); gewonnen ist damit allerdings auch nichts. Im Grunde handelt es sich um eine Aufgabe, die der Rechtswissenschaft seit jeher obliegt.

13 Eine gänzlich andere Beschreibung des Aufgabenfelds der Verwaltungsrechtswissenschaft ist bei den Verfechtern des steuerungswissenschaftlichen Ansatzes zu finden; siehe dazu statt vieler Voßkuble, Verwaltungsrechtswissenschaft (Fn. 7), $\mathbb{1}$ Rn. 14. Näher dazu unten: C.I.

14 Gegenstand der Wissenschaft vom Verwaltungsrecht ist demnach nicht die Verwaltung, sondern die Summe jener rechtlichen Normen, nach denen sich diese vollzieht; Winkler, Verwaltungsrecht (Fn. 6), S. 5; anders jedoch von Arnauld, Wissenschaft (Fn. 4), S. 71; R. Breuer, Zur Lage der deutschen Verwaltungsrechtswissenschaft, Die Verwaltung 36 (2003), S. $285 \mathrm{ff}$.

15 Jestaedt, Disziplin (Fn. 4), S. 278 f. 
ziert: ${ }^{16}$ dies jedoch kann nur die Wissenschaft vom Verwaltungsrecht sein. Dieses spezielle Erkenntnisinteresse lässt sich nicht hinterfragen, ohne zugleich das Recht in seiner Eigenschaft als solches in Zweifel zu ziehen. ${ }^{17}$ Das Tätigkeitsfeld der Publizistik (wie man die Wissenschaft vom öffentlichen Recht mitunter bezeichnet) ${ }^{18}$ korrespondiert nach Art, Umfang und Dauer unmittelbar mit der Reichweite des Rechts im Bereich der Verwaltung: die Beziehungen des einzelnen zur hoheitlichen Gewalt bildeten erst von jenem Moment an ein Thema der Rechtswissenschaften, als diese Verhältnisse verrechtlicht wurden bzw. als rechtlich erkannt worden sind ${ }^{19}$ - diesen Zeitpunkt markiert die Entstehung des Rechtsstaats. War es nicht eine der größten Leistungen der modernen Rechtswissenschaft, auch jene Normen, die heutzutage das Recht der Verwaltung ausmachen, in ihrer Eigenschaft als geltendes Recht erkannt und behandelt zu haben? ${ }^{20}$ Von einer Geltung rechtlicher Normen im Bereich der Verwaltung ist nur dann und ist nur insoweit zu sprechen, wie es gelingt, den Fundus rechtlicher Regeln von sämtlichen anderen Maßstäben zu unterscheiden, die an das verwaltungsbehördliche Handeln angelegt werden können. ${ }^{21}$

Gemessen an diesen Prämissen, hat man es nur dort mit einer rechtswissenschaftlichen Herangehensweise an die rechtliche Ordnung zu tun, wo sich das Streben nach Erkenntnissen auf dieses Entweder-Oder von Rechtmäßigkeit und Rechtswidrigkeit

16 Anders als dies oft gesagt wird, kommt es hierbei nicht darauf an, ob man im Verwaltungsrecht ein „Kontrollprogramm“, ein „Verhaltensprogramm“ oder „Programm zur Lösung von Problemen durch administratives Handeln" erblickt (alle Begriffe bei W. Hoffmann-Riem, Methoden einer anwendungsorientierten Verwaltungsrechtswissenschaft, in: Schmidt-Aßmann/Hoffmann-Riem [Hrsg.], Methoden der Verwaltungsrechtswissenschaft, Baden-Baden 2004, S. 9 [46]) - von der Funktion des Verwaltungsrechts her gesehen ist jede dieser Bezeichnungen richtig. $\mathrm{Zu}$ bestreiten ist allerdings, dass eine solche funktionalistische Betrachtung und Unterscheidung für das juristische Denken überhaupt relevant ist, da dieses gerade nicht nach den Funktionen des Rechts fragt, sondern darin eine autonome und absolute Größe erkennt.

17 Dies beantwortet auch die von K. Schneider (Zur Eignung des Forschungsprogramms der Reinen Rechtslehre als Wissenschaftstheorie einer Wissenschaft vom Öffentlichen Recht, in: Funke/Lüdemann [Hrsg.], Öffentliches Recht und Wissenschaftstheorie, Tübingen 2009, S. 45 [48]) aufgeworfene Frage, woher die Rechtswissenschaftstheorie ihr Wissen über das richtige Ziel der Rechtswissenschaft nimmt: es ergibt sich daraus, dass es sich bei der Rechtswissenschaft um die auf das Recht (in seiner Eigenschaft als solches) bezogene Wissenschaft handelt.

18 So Jestaedt, Disziplin (Fn. 4), S. 243 (und öfter).

19 Vgl. dazu ausführlich H. Bauer, Verwaltungsrechtslehre im Umbruch?, Rechtsformen und Rechtsverhältnisse als Elemente einer zeitgemäßen Verwaltungsrechtsdogmatik, Die Verwaltung 25 (1992), S. 301 (317f.); Breuer, Verwaltungsrechtswissenschaft (Fn. 14), S. 275 ff. und M. Stolleis, Entwicklungsstufen der Verwaltungsrechtswissenschaft, in: Hoffmann-Riem/Schmidt-Aßmann/Voßkuhle (Hrsg.), Grundlagen des Verwaltungsrechts, 2. Aufl., München 2012, Bd. I, $\$ 2$ Rn. 47.

20 Bei der (oft kritisch kommentierten) Konzentration auf die gerichtliche Kontrollperspektive handelt es sich deshalb nicht um eine (die Rechtswissenschaft hemmende) Verkürzung der Dinge, sondern um das Hervortreten echter Jurisprudenz für das Recht der Verwaltung. Tendenziell anders jedoch G. Püttner, Entwicklungstendenzen in der Dogmatik des Verwaltungsrechts, in: FS Bachof, München 1984, S. 115 (127). Siehe dazu auch nachstehend: C.I.

21 Der häufig bemerkte Bedeutungsverlust der juristischen Dogmatik im Bereich des Verwaltungsrechts weist somit letztlich auf den Bedeutungsverlust des Verwaltungsrechts selbst hin. Vgl. dazu H. SchulzeFielitz, Einheitsbildung durch Gesetz oder Pluralisierung durch Vollzug, in: Trute/Groß/Röhl/Möllers (Hrsg.), Allgemeines Verwaltungsrecht - zur Tragfähigkeit eines Konzepts, Tübingen 2008, S. 135 (158f.). 
richtet. Mit dem Forschungsgegenstand Recht kann man sich freilich unter einer Vielzahl weiterer Erkenntnisziele befassen - wer dies tut, betrachtet das Recht indes nicht als eigenständige, absolut zu denkende Größe (genau das ist es nämlich, was die juristische Sicht definiert), sondern erfasst das Recht als historisches Datum, als gesellschaftliches Phänomen, als ökonomischen Faktor oder als psychologisch zu untersuchendes Entscheidungskriterium. ${ }^{22}$ Erfreulicherweise wagt bislang noch kein Theoretiker den Gedanken, dass eine derartige Wissenschaft, die das Recht als einen solch fundamentalen und autonomen Maßstab des menschlichen Handelns erkennt und ein begründbares Entweder-Oder von Rechtmäßigkeit und Rechtswidrigkeit daraus entwickelt, nicht mehr gebraucht wird ${ }^{23}$ - doch liegt nicht genau diese Folge in der Konsequenz einer schrittweise sich vollziehenden Preisgabe der Prämissen eines originär juristischen Denkens, wie sie uns im Verwaltungsrecht im wachsenden Maße begegnet? Wenn man das spezifische Erkenntnisinteresse der Wissenschaft vom Verwaltungsrecht in der Feststellung von Rechtmäßigkeit und Rechtswidrigkeit sieht, wird man - das sei an diesem Punkt noch erwähnt - auch die Behauptung zurückweisen müssen, dass erst die Erkenntnismethode den Erkenntnisgegenstand der Verwaltungsrechtswissenschaft konstituiert. ${ }^{24}$ Methoden, die dazu anleiten, Rechtmäßigkeit und Rechtswidrigkeit zu unterscheiden, beziehen sich eo ipso auf den Erkenntnisgegenstand Recht; andersherum betrachtet sind es per definitionem allein die Methoden des rechtswissenschaftlichen Denkens, die aus einem solchen Erkenntnisinteresse heraus auf den Erkenntnisgegenstand Recht gerichtet sein können. ${ }^{25}$ Dies jedoch heißt: Erkenntnisgegenstand und Erkenntnismethoden sind im Falle der Rechtswissenschaft zwangsläufig aufeinander fixiert. ${ }^{26}$

\section{Schwierigkeiten und Schlussfolgerungen, die sich aus einer solchen Bestimmung des rechtswissenschaftlichen Erkenntnisinteresses ergeben}

Wenn man das Erkenntnisinteresse der Verwaltungsrechtswissenschaft in dieser Weise von der Eigenart des Juristischen her konstruiert, ergibt sich bei näherer Betrachtung die Frage, ob es tatsächlich diese Unterscheidung von Rechtmäßigkeit und Rechtswidrigkeit selbst ist, der sich die Rechtswissenschaft zuwenden muss, oder ob

22 Ähnlich Jestaedt, Disziplin (Fn. 4), S. 271; vgl. auch von Arnauld, Wissenschaft (Fn. 4), S. 68.

23 W. Pauly, Wissenschaft vom Verwaltungsrecht: Deutschland, in: von Bogdandy/Cassese/Huber (Hrsg.), Handbuch Ius Publicum Europaeum, Bd. IV, Heidelberg 2011, 58 Rn. 22; vgl. auch H.- $H$. Trute, Die Wissenschaft vom Verwaltungsrecht: Einige Leitmotive zum Werkstattgespräch, in: Die Wissenschaft vom Verwaltungsrecht (Die Verwaltung, Beiheft 2), Berlin 1999, S. 9, (13).

24 So Jestaedt, Disziplin (Fn. 4), S. 268; ders., Wissenschaftstheorie (Fn. 4), S. 37. Kritisch demgegenüber Möllers, Wissenschaftstheorie (Fn. 4), S. 160.

25 Siehe dazu Jestaedt, Disziplin (Fn. 4), S. 272 und Möllers, Wissenschaftstheorie (Fn. 4), S. 160.

26 Vgl. dazu auch Jestaedt, Disziplin (Fn. 4), S. 263 f.; Winkler, Verwaltungsrecht (Fn. 6), S. 9 f. sowie Schneider, Wissenschaftstheorie (Fn. 17), S. 52 f. (dessen Darstellung deutlich die epistemologischen Abgründe und Aporien zeigt, zu denen man zwangsläufig gelangt, wenn man den Bezug der Rechtswissenschaft auf die Unterscheidung von Rechtmäßigkeit und Rechtswidrigkeit aus den Augen verliert). 
ihr zentrales Erkenntnisziel nicht vielmehr darauf beschränkt ist, Methoden zu definieren, anhand derer ein Jurist, der das Recht anwenden soll, Rechtmäßigkeit und Rechtswidrigkeit zu bestimmen vermag. Die richtige Antwort auf diese Frage muss lauten, dass beide Aspekte zu den Aufgaben der Rechtswissenschaft zählen. Im ersten Fall (wenn also das Erkenntnisziel in der Unterscheidung von Rechtmäßigkeit und Rechtswidrigkeit im Hinblick auf konkrete Konfliktlagen und hierdurch aufgeworfene Rechtsfragen liegt) benötigt die Jurisprudenz ihrerseits ein Arsenal an Methoden, welches dazu verhilft, Geltung und Inhalt der rechtlichen Norm zu ermitteln. Indem sie vom Boden einer konkreten Rechtsordnung aus operiert, besitzt sie im Regelfall ein solches Rüstzeug, ${ }^{27}$ dass dieses Arsenal in verschiedenen Rechtsordnungen unterschiedlich beschaffen sein mag (genauer gesagt: dass Juristen, die im Rechtssystem des angelsächsischen Common Law tätig sind, gänzlich andere Methoden der Rechtsfindung anwenden als die Juristen in Frankreich oder in Deutschland), könnte ihr grundsätzlich gleichgültig sein. Im zweiten Fall (wenn also das Erkenntnisinteresse des Rechtswissenschaftlers gerade darin besteht, überhaupt erst die Methoden zu definieren, anhand derer man zwischen Rechtmäßigkeit und Rechtswidrigkeit zu unterscheiden vermag) gelangte man aus methodologischer Sicht zu der kniffligen Frage, welche regelhaften Herangehensweisen es gibt, die dazu führen, passende Methoden für die Erkenntnis des Rechtsinhalts zu definieren. Ob sich eine solche Art von Metamethodik (die uns verrät, wie man die Methoden der Rechtsfindung ihrerseits identifiziert) überhaupt ausdenken lässt, ${ }^{28}$ erscheint indes fraglich - macht nicht gerade die Angestrengtheit der neueren methodologischen Reflexionen vor allem das eine bewusst: dass nämlich für die Feststellung der Rechtserkenntnismethoden keine methodischen Regeln bestehen ${ }^{29}$ Wenn die neuere Erkenntnistheorie davon ausgeht, dass Methoden ihrerseits eher Festlegungen und Dezisionen als Erkenntnisse sind, ${ }^{30}$ so spricht sie damit eine Annahme aus, die man im Bereich der Rechtswissenschaft seit jeher als Selbstverständlichkeit ansehen durfte. Und stellt nicht genau dieser Umstand den Hintergrund der gelegentlich anzutreffenden Forderung dar, über Methoden sei nicht zu sprechen ${ }^{31}$ - die von dieser Warte her wenn schon nicht zu billigen, so zumindest jedoch leichter nachzuvollziehen sein sollte?

27 Jestaedt, Disziplin (Fn. 4), S. 272; C. Waldhoff, Kritik und Lob der Dogmatik, Rechtsdogmatik im Spannungsfeld von Gesetzesbindung und Funktionsorientierung, in: Kirchhof/Magen/Schneider (Hrsg.), Was weiß Dogmatik?, Tübingen 2012, S. 17 (20); siehe ferner auch Möllers, Wissenschaftstheorie (Fn. 4), S. 160 f. Ob man davon sprechen soll, dass der Methodenwahl „Verfassungsrang“ zukommt (so B. Rüthers, Methodenrealismus in Jurisprudenz und Justiz, JZ 2006, S. 53 [60]), mag hier dahingestellt bleiben.

28 Bejahend Hoffmann-Riem, Methoden (Fn. 16), S. 19.

29 Auch das Postulat: „Rechtswissenschaft ist methodisch oder sie ist nicht“ (so Voßkuble, Pragmatik [Fn. 2], S. 172 f.) ist daher wohl insoweit nicht erfüllbar, als es um jene Meta-Ebene geht, auf der die Methoden der Rechtsauslegung festgestellt werden.

30 Schneider, Wissenschaftstheorie (Fn. 17), S. 59.

31 Siehe dazu Hoffmann-Riem, Methoden (Fn. 16), S. 11 (m.w.N.) und Voßkuble, Pragmatik (Fn. 2), S. 176. 
Mit dieser theoretischen Differenzierung zwischen einer (den rechtsordnungsimmanenten Methoden der Rechtserkenntnis verpflichteten) Bereitstellung eines konkreten Rechtmäßigkeitsurteils einerseits und einer (gezwungenermaßen methodenlos zu Werke gehenden) Bemühung um die Entwicklung entsprechender Rechtserkenntnismethoden andererseits hängt die Unterscheidung von Rechtsdogmatik ${ }^{32}$ und juristischer Methodenlehre zusammen (ohne dass sie hiermit gleichgesetzt werden dürfte). Wie kompliziert es erscheint, Dogmatik und Methodenlehre auseinanderzuhalten, bezeugt der Versuch, zwischen einer sogenannten Rechtsquellenlehre (als einem Bestandteil der Rechtsdogmatik) und einer sogenannten Rechtsgewinnungslehre (als einem Bestandteil der Rechtsmethodik) zu differenzieren. ${ }^{33}$ Beide betreffen dasselbe zentrale Problem, wie das Recht als solches erkannt werden kann. Für denjenigen, der dazu bereit ist, das verwaltungsjuristische Denken auf die Unterscheidung von Rechtmäßigkeit und Rechtswidrigkeit zu konzentrieren, bildet die Suche nach einer theoretischen Grenze zwischen Rechtsdogmatik und Methodenlehre indes eine nachrangige und nebensächliche Frage, weil es für die Probleme, mit denen er konfrontiert ist, hierauf nicht wesentlich ankommt - indem er den Inhalt des Rechts zu bestimmen hat, betreibt er beides zugleich. Die Ursache hierfür ist leicht zu erklären: dogmatische Sätze über den Inhalt des Rechts sind nur dann als solche anzuerkennen, wenn sie methodengerecht begründbar erscheinen. Weil die Bestimmung dieser Methodik (als ein Charakteristikum der konkreten rechtlichen Ordnung) ${ }^{34}$ gleichfalls in die Hände der Juristen gelegt ist, lässt sich behaupten, dass die Dogmatik des Rechts zur Rekonstruktion der Methodik mindestens so viel beiträgt, wie andersherum die Rechtsmethodik zur Beherrschung der Rechtsdogmatik; bei der Arbeit mit der rechtlichen Norm, die zur Erkenntnis von Rechtswidrigkeit oder Rechtmäßigkeit führen soll, vollziehen sie sich im Regelfall uno actu. Ein gewisses dogmatisches Rechtsquellenverständnis liegt der Methodik immer ein Stück weit voraus: der Jurist muss zunächst annehmen, dass dieser und jener Typus von Sätzen Gesetze im rechtlichen Sinne darstellt (oder auch: er muss annehmen, dass es so etwas wie Gewohnheitsrecht gibt), bevor er sich der Frage zuwenden kann, mit welchen Mitteln er den Inhalt der Normen identifiziert und seine Rechtsentscheidung begründet. Es wäre interessant zu erforschen (doch es zählt nicht zum Gegenstand dieses Essays), inwiefern sich eben dieses Wechselspiel gegenwärtig im Recht der EU beobachten lässt. Auch dort gilt, dass sich die Juristen in einer konkreten rechtlichen Ordnung sowohl über die

32 Ausführlich zu verschiedenen Definitionen der Rechtsdogmatik Waldhoff, Rechtsdogmatik (Fn. 27), S. $22 \mathrm{ff}$.

33 Siehe dazu Jestaedt, Disziplin (Fn. 4), S. 275 (dort in Fn. 172).

34 Näher dazu S. Haack, L'État - qu'est-ce que c'est? - Die Wissenschaft vom öffentlichen Recht und ihre Methoden nach sechzig Jahren Arbeit mit dem Grundgesetz, Der Staat 49 (2010), S. 107 (121 ff.); ders., Primitive Staatstheorie, Der Staat 51 (2012), S. 57 (75 f.). 
Rechtsdogmatik als auch über die Rechtsmethodik erfolgreich verständigen müs$\operatorname{sen}^{35}$ - und manche halten es offenbar wiederum für ideal, über die Methodik (welche leicht in die erwähnten epistemologischen Abgründe führt) so wenig wie möglich zu sprechen. Kurzum: Rechtsmethodik und Rechtsdogmatik erscheinen in jedem rechtswissenschaftlichen Denken, das auf die Unterscheidung von Rechtswidrigkeit und Rechtmäßigkeit abzielt, auf das Engste miteinander verbunden. ${ }^{36}$

Wenn man das Erkenntnisinteresse der Verwaltungsrechtswissenschaft in der Anleitung zur Unterscheidung von Rechtmäßigkeit und Rechtswidrigkeit (hinsichtlich des Rechts der Verwaltung) erblickt, erweist sich letztendlich auch die in der theoretischen Literatur ${ }^{37}$ anzutreffende Differenzierung zwischen der sogenannten Teilnehmerperspektive und der sogenannten Beobachterperspektive als problematisch. Eine Rechtswissenschaft, deren Aufgabe darin besteht, dem Rechtsanwender beizubringen, wie man Rechtmäßigkeit und Rechtswidrigkeit unterscheidet, lässt sich nicht ohne weiteres als Teilnehmerin an einer konkret-sachbezogenen juristischen Auseinandersetzung begreifen. Ebensowenig befindet sie sich in der Rolle einer Beobachterin, welche sich die Arbeit des juristischen Betriebes von außen her ansieht (dies zu tun, hieße nämlich sozialwissenschaftlichen anstatt rechtswissenschaftlichen Erkenntniszielen zu folgen). In der gleichen Weise, wie jene Personen, die bei einem Fußballspiel anwesend sind, nicht restlos unter die Begriffe des Teilnehmers und des Beobachters subsumiert werden können (es finden sich daneben Schiedsrichter, Trainer und eine Vielzahl von Helfern), lassen sich auch die in der Arena der verwaltungsrechtlichen Arbeit zu besetzenden Rollen nicht auf Teilnahme und Beobachtung

35 Eine Rechtswissenschaft ohne jede Form von Dogmatik lässt sich daher kaum denken - der Stellenwert der Dogmatik im juristischen Denken der Wissenschaft und der Praxis schwankt indes ganz erheblich; vgl. Waldhoff, Rechtsdogmatik (Fn. 27), S. 31 und 36 f.; anders jedoch Lepsius, Dogmatik (Fn. 4), S. 46 (der Dogmatik als etwas spezifisch Deutsches und nichts der Rechtswissenschaft Immanentes betrachtet); ähnlich Jestaedt, Kommunikationsformat (Fn. 4), S. 118; ders., Disziplin (Fn. 4), S. 247.

36 Vgl. Waldhoff, Rechtsdogmatik (Fn. 27), S. 27 (der die Auslegungsmethoden zur Dogmatik hinzuzählt); siehe auch im selben Band den Beitrag von Lepsius, Dogmatik (Fn. 4), S. 42; Jestaedt, Wissenschaftstheorie (Fn. 4), S. 35.

37 Siehe von Arnauld, Wissenschaft (Fn. 4), S. 69 f. und Jestaedt, Disziplin (Fn. 4), S. 264. 
reduzieren. ${ }^{38}$ Die Perspektive der Rechtswissenschaft sollte man insofern am ehesten als eine solche der spezifischen, rechtsbezogenen Anleitung bezeichnen. ${ }^{39}$

\section{Vier Schritte zu einer Läuterung des verwaltungsrechtstheoretischen Denkens}

\section{Die Beschränkung einer rechtspolitischen Inanspruchnahme der juristischen Arbeit}

Ausgehend von dieser Konzentration des verwaltungsjuristischen Denkens auf das, was den eigentümlichen Kern der Wissenschaft vom Verwaltungsrecht ausmacht, soll in den folgenden Abschnitten eine Reihe von Fehlentwicklungen aufgezeigt werden, die gegenwärtig dazu beitragen, das rechtswissenschaftliche Denken im Bereich des Verwaltungsrechts in Frage zu stellen. Hierbei handelt es sich um das Vermischen der juristischen Methodik mit dem Bestreben der Rechtspolitik (1.), um ein unreflektiertes Ineinandergreifen von Rechts- und Sozialwissenschaften (2.), um das Überbewerten von Bedürfnissen der verwaltungsjuristischen Praxis (3.) sowie schließlich um das Missverständnis und die Überschätzung der historischen Bedingtheit rechtswissenschaftlicher Arbeit (4.). Zu selten thematisiert die Wissenschaft vom Verwaltungsrecht zunächst das Problem (denn um ein solches handelt es sich), was der politische Zusammenhang, in dem die gesetzliche Regelung steht, für die juristische Arbeit bedeutet. ${ }^{40}$ Dass man um die Beschäftigung mit den politischen Zielen des Gesetzgebers im Hinblick auf die Ermittlung des Zwecks einer Norm nicht umhin kommt, steht außer Frage. Unbedingt zu beanstanden wäre es jedoch, wenn über den begrenzten Einsatzbereich teleologischer Auslegungsargumente hinaus Rechtspolitik

38 Appel, Steuerungswissenschaft (Fn. 4), S. 234 (dort in Fn. 20); vgl. auch von Arnauld, Wissenschaft (Fn. 4), S. 69 f. und Möllers, Wissenschaftstheorie (Fn. 4), S. 167.

39 Die Verbindung von Rechtswissenschaft und Rechtspraxis in einem gemeinsamen Diskurs stellt - anders als Lepsius (Dogmatik [Fn. 4], S. 60) behauptet - keine Gefahr dar für den Wissenschaftscharakter der Rechtswissenschaft (siehe dazu auch E. Schmidt-Aßmann, Methoden der Verwaltungsrechtswissenschaft - Perspektiven der Systembildung, in: Schmidt-Aßmann/Hoffmann-Riem [Hrsg.], Methoden der Verwaltungsrechtswissenschaft, Baden-Baden 2004, S. 387 [389]). Ist diese schroffe Entgegensetzung von „Wissenschaft“ und „Praxis“ nicht selbst - weit über den Bereich der Rechtswissenschaften hinaus - ein theoretisch zweifelhaftes und praktisch immer wieder in Frage gestelltes Konstrukt, das trennen will, was letztendlich zusammengehört? Wissenschaftlichkeit bildet das Kennzeichen einer bestimmten Herangehensweise, unabhängig davon, wo diese sich findet; den Rechtspraktiker oder den Privatgelehrten kann sie ebenso auszeichnen, wie sich Universitätsprofessoren anzeigen ließen, denen sie abhanden gekommen erscheint (zur wissenschaftlichen Qualität der Forschung in der deutschen Staats- und Verwaltungsrechtswissenschaft ausführlich H. Schulze-Fielitz, Was macht die Qualität öffentlich-rechtlicher Forschung aus?, JÖR 50 [2002], S. 1). Als bedenklich erweist sich insofern nicht so sehr der gemeinsame Diskurs von Vertretern der Rechtswissenschaft und der Rechtspraxis (den andere Wissenschaftszweige in vergleichbarer Form kennen - man denke nur an die Forschung in Industrieunternehmen), sondern die einseitige Ausrichtung der Theorie an den vermeintlichen Erfordernissen der Praxis; s. dazu unten: C.III.

40 Allgemein zum Verhältnis von Rechtsdogmatik und Rechtspolitik H. Schulze-Fielitz, Verwaltungsrechtsdogmatik als Prozess der Ungleichzeitigkeit, Die Verwaltung 3 (1994), S. 277 (299) und Winkler, Verwaltungsrecht (Fn. 6), S. 29. Für eine größere Distanz der Wissenschaft vom öffentlichen Recht gegenüber der Politik plädiert Schulze-Fielitz, Qualität (Fn. 39), S. 62. 
in das Rechtsdenken einfließt. ${ }^{41}$ Dass dies in immer größerem Umfang geschieht, ${ }^{42}$ ließe sich an zahllosen Beispielsfällen belegen. Nicht selten wird die Handhabung öffentlich-rechtlicher Normen nach dem rechtspolitischen Belieben des Rechtsanwenders offen bekundet ${ }^{43}$ - mitunter ruft man sogar dazu auf, dies noch offensiver als bisher zu praktizieren. ${ }^{44}$ Dies gilt - wie ausdrücklich betont werden muss - gleichermaßen für den Einfluss der rechtspolitischen Ambitionen auf das juristische Denken in der Wissenschaft wie in der Praxis; dem Anliegen dieses Essays entsprechend soll es hier jedoch vorrangig um die Beeinflussung des Rechtsdenkens in der Wissenschaft gehen. Um den juristischen Charakter der Handhabung verwaltungsrechtlicher Vorschriften aufrechtzuerhalten und den wissenschaftlichen Anspruch hierbei nicht zu verfehlen, zählt es zu den wichtigsten Aufgaben der verwaltungsrechtstheoretischen Arbeit, politisch motivierten Zugriffen auf die Rechtsanwendung Schranken zu setzen und ihnen den Platz zuzuweisen, der ihnen zusteht. Zu finden ist dieser Platz überall dort, wo das Recht (politische) Entscheidungsspielräume eröffnet. Auf die Zurückhaltung, die vom Juristen an den entsprechenden Stellen verlangt werden muss, ist sogleich zu sprechen zu kommen; um so stärker und um so selbstbewusster hat er jedoch sein juristisches Denken zu präsentieren (und hat er hierdurch die juristische Weltsicht zu repräsentieren), wo es darum geht, diese Grenzen von einer rechtlichen Warte her abzustecken und zu kontrollieren. Obgleich noch keine Art von rechtlichem Denken hervorgebracht wurde, die gegen den politischen Missbrauch gefeit ist, sollte es doch das Anliegen der Rechtswissenschaft sein (dies nicht zuletzt im Interesse ihres eigenen Wertes und ihres Daseins als solche), die Anleitungen zur Unterscheidung von Rechtmäßigkeit und Rechtswidrigkeit so zu konzipieren, dass sie diese Anfälligkeit nicht von sich aus vervielfacht, sondern so weit wie möglich verringert. ${ }^{45} \mathrm{Um}$ ein entsprechendes Missverständnis nicht zu provozieren, sei an dieser Stelle betont, dass es hier keineswegs darum geht, die Tätigkeit der Verwaltung selbst als unpolitisch zu qualifizieren, wie dies früher - zum Teil wohl aufgrund antidemokratischer Ressentiments - versucht worden ist; ${ }^{46}$ worauf es uns in diesem Zusammenhang ankommt, ist vielmehr eine strikte gedankliche Trennung von juristischem und politischem Denken im Umgang mit verwaltungsrechtlichen

41 Anders jedoch Möllers, Wissenschaftstheorie (Fn. 4), S. 172; siehe dazu auch Lepsius, Dogmatik (Fn. 4), S. 58.

42 Vgl. auch Kahl, Verwaltungsrecht (Fn. 10), S. 469 und Voßkuble, Verwaltungsrechtswissenschaft (Fn. 7), $\$ 1$ Rn. 12.

43 Bedenklich weit geht z. B. Voßkuble, Pragmatik (Fn. 2), S. 180, 192 f.

44 Vgl. dazu auch Lepsius, Dogmatik (Fn. 4), S. 58; Möllers, Wissenschaftstheorie (Fn. 4), S. 172.

45 Dass rechtswissenschaftliche Aussagen immer auch politische Bedeutung besitzen (so der Sache nach Möllers, Wissenschaftstheorie [Fn. 4], S. 172), besagt nicht ohne weiteres, wie die Wissenschaft hiermit richtigerweise umgehen sollte. Aus den Gründen, die im Haupttext ausgeführt werden, hat sich diese darum zu bemühen, die politischen Gehalte ihrer eigenen wissenschaftlichen Sätze soweit wie möglich unbeachtet zu lassen.

46 Siehe dazu F. Günther, Denken vom Staat her, München 2004, S. 86 (136 f., 260 m.w.N. [u.a. zu E. Forsthoff]). 
Normen. ${ }^{47}$ Überall dort, wo Spielräume bleiben für die politische Dezision, sind diese (unabhängig davon, ob es die Tätigkeit von Verfassungsorganen oder die Tätigkeit von Verwaltungsstellen betrifft) als solche auszuweisen; die politische Dezision als juristische Deduktion zu verbrämen, trägt in erheblichem Umfang zur Beliebigkeit und zur Entwertung des Rechtsdenkens bei. Gerade dort, wo das Recht Beurteilungsspielräume, Einschätzungsprärogativen oder Verwaltungsermessen bereitstellt, sind rechtliche Norm und politische Ziele strikt zu unterscheiden. Aus juristischer Sicht ist dabei zu beachten, dass es die rechtliche Norm ist, die diese Spielräume festlegt. Beispiele hierfür bilden die Ausgestaltung des Staatsangehörigkeitsrechts ${ }^{48}$ oder die Dogmatik der staatlichen Schutzpflichten ${ }^{49}$ in den verschiedenen Bereichen der Verwaltung riskanter Technologien. ${ }^{50}$ Eine Läuterung der Verwaltungsrechtstheorie, wie dieser Essay sie anregen soll, läuft hier und in ähnlichen Konstellationen darauf hinaus, politische Entscheidungsspielräume, die von Gesetzes wegen bestehen, möglichst weit geöffnet zu halten. ${ }^{51}$

Gänzlich anders hat sich hierzu jedoch die Neue Verwaltungsrechtswissenschaft positioniert, wenn diese der hergebrachten juristischen Sichtweise vorwirft, zu stark auf die Perspektive der gerichtlichen Kontrolle ausgerichtet zu sein, und sich darum bemüht, Maßstäbe für eine Ausfüllung von Entscheidungsspielräumen zu liefern. ${ }^{52}$ Sofern es sich bei den von ihr hervorgebrachten Kriterien um rechtliche Maßstäbe handelt, wäre an sich nichts dagegen zu sagen ${ }^{53}$ - außer vielleicht, dass in derartigen Fällen letztendlich doch kein Entscheidungsspielraum bestünde und die Neue Verwaltungsrechtswissenschaft insofern eigentlich nichts tut, was wirklich neu ist. ${ }^{54}$ Auch der Umstand, dass es schwierig sein kann zu erkennen, ob bestimmte Prinzipien (wie z.B. das Effizienzgebot) als politische Handlungsmaximen oder als rechtliche Grundsätze gelten, vermag daran nichts zu ändern: dies zu untersuchen, ist seit jeher

47 Gegen eine Verschleierung politischer Argumente durch Rechtsdogmatik auch Lepsius, Dogmatik (Fn. 4), S. 58; ähnlich S. Augsberg, Die aktuelle Methodendiskussion: eine wissenschaftstheoretische Renaissance?, in: Funke/Lüdemann (Hrsg.), Öffentliches Recht und Wissenschaftstheorie, Tübingen 2009, S. 145 (182); siehe hierzu außerdem Winkler, Verwaltungsrecht (Fn. 6), S. 34.

48 Näher dazu S. Haack, Staatsangehörigkeit - Unionsbürgerschaft - Völkerrechtssubjektivität in: Isensee/Kirchhof (Hrsg.), Handbuch des Staatsrechts, 3. Aufl., Heidelberg 2012, Bd. X, $\mathbb{2 0 5}$ Rn. 9.

49 Siehe dazu die kritische Einschätzung von Lepsius, Dogmatik (Fn. 4), S. 58.

50 Näher hierzu S. Haack, Grundrechtsschutz und Risikoprävention, Eine verfassungsrechtliche Skizze, in: Jaeckel/Janssen (Hrsg.), Risikodogmatik im Umwelt- und Technikrecht, Tübingen 2012, S. 21 (29 ff.).

51 In eine ähnliche Richtung geht Möllers, Methoden (Fn. 2), $\mathbb{3}$ Rn. 6.

52 Siehe dazu Appel, Steuerungswissenschaft (Fn. 4), S. 260; Schmidt-Aßmann, Ordnungsidee (Fn. 7), S. 26 und Voßkuble, Verwaltungsrechtswissenschaft (Fn. 7), $\$ 1$ Rn. 14.

53 Vgl. von Arnauld, Wissenschaft (Fn. 4), S. 97 f. und K. Waechter, Diskussionsbeitrag, VVDStRL 67 (2008), S. 345 (346). Wie diese Unterscheidung (des rechtlichen Maßstabs von allen anderen Maßstäben des behördlichen Handelns) bei vielen Vertretern des steuerungswissenschaftlichen Ansatzes und der „rechtswissenschaftlichen“ Governance-Forschung verschwimmt, ist u. a. bei Franzius, Governance (Fn. 8), S. 218 f. erkennbar.

54 Siehe dazu auch von Arnauld, Wissenschaft (Fn. 4), S. 98. 
ein originäres Geschäft des Juristen (insbesondere des Verfassungsjuristen) - man denke hierbei nur an die Entwicklung von Prinzipien des Allgemeinen Verwaltungsrechts vor dessen Kodifizierung. ${ }^{55} \mathrm{Um}$ rechtliche Maßstäbe kann es sich dabei nur unter der Voraussetzung handeln, dass entsprechende Inhalte einer rechtlichen Norm anhand der konsentierten Interpretationsmethoden begründbar erscheinen.

Indem die Neue Verwaltungsrechtswissenschaft dazu auffordert, man solle die Perspektive der Verwaltung einnehmen, wenn es um das Ausfüllen von Handlungsspielräumen geht, ${ }^{56}$ rückt sie von einer weiteren Prämisse des Rechtsdenkens ab (über welche in der Auseinandersetzung mit diesem Ansatz bislang zu wenig gesagt worden ist): gemeint ist hiermit die Objektivität der rechtlichen Ordnung und damit zugleich die Neutralität des juristischen Prüfens. Diese Objektivität ist unmittelbar mit dem Wesen rechtlicher Normen verbunden: ungeachtet der politischen Motive seines Erlasses kennzeichnet es die Funktionsweise des Rechts, dass sich an objektive Tatbestandsvoraussetzungen festgelegte Rechtsfolgen knüpfen. Das Gesetz muss unabhängig davon, welchen konkreten Sachverhalt man darunter zu subsumieren hat und wer mit der Sache befasst ist, ein und dasselbe besagen. Weil es sich hierbei nur um ein Ideal handeln kann, ließe sich insoweit auch von einem Neutralitätsideal des Rechtsdenkens sprechen: diese Gleichgültigkeit des Gesetzes gegenüber sämtlichen Normadressaten und dieser Abstand zu den Konflikten, für deren rechtliche Lösung es den Maßstab bereitstellt, bilden ein Essential jedes originären juristischen Denkens. Am Leitbild des richterlichen Entscheidens orientiert sich die juristische Methodik insbesondere deshalb, weil eben dieser grundlegende Objektivitätsanspruch des Rechts bei der Arbeit des Richters am klarsten hervortritt (obgleich er nicht hierauf beschränkt ist, sondern bei jeder rechtlichen Prüfung im Raum steht). ${ }^{57}$ Auch die häufig kritisierte Fokussierung der Juristenausbildung auf das Berufsbild des Richters findet hierin ihre Rechtfertigung. Dass die Anwendung des Verwaltungsrechts dazu dient, politische Ziele zu realisieren, erlaubt und erfordert es keineswegs, die Objektivität des Rechts als Prämisse zu eliminieren und deren Rang in der Verwaltungsrechtstheorie an die politische Zweckgerichtetheit zu vergeben. ${ }^{58}$ Gerade das ist jedoch ein (bislang zu selten erhobener) Einwand, welcher gegen die Neue Verwaltungsrechtswissenschaft vorgebracht werden muss: anstelle der Objektivität von

55 Eine Entwicklung, die in Deutschland vor dem Inkrafttreten des VwVfG zu beobachten war und die sich derzeit im EU-Recht vollzieht; vgl. von Arnauld, Wissenschaft (Fn. 4), S. 76 f.

56 So Schmidt-Aßmann, Ordnungsidee (Fn. 7), S. 18 (26, 29); ders., Methoden (Fn. 39), S. 405.

57 Auch die Kritik an einer (missverstandenen) Konzentration auf den Rechtsschutz besitzt Tradition siehe hierfür beispielsweise P. Häberle, Die Verfassung des Pluralismus, Königstein/Ts. 1980, S. 285 (dort in Endnote 130); Park, Rechtsfindung (Fn. 11), S. 292 sowie Püttner, Entwicklungstendenzen, S. 127 (dieser jedoch eher referierend als kritisierend). Dass sie bislang nichts bewirkt hat, könnte aus der Sicht der hier vorzutragenden Lehren darauf beruhen, dass hinter dieser Konzentration auf den Rechtsschutz das rechtswissenschaftliche Denken und der Rechtscharakter des Verwaltungsrechts stehen.

58 Vgl. dazu auch W. Henke, Wandel der Dogmatik des öffentlichen Rechts, JZ 1992, S. 541 (547). 
rechtlichen Normen und der Neutralität des juristischen Denkens erhebt sie den instrumentalen Charakter des Verwaltungsrechts für politische Zwecke zur zentralen Maxime der verwaltungsjuristischen Arbeit; ${ }^{59}$ dies jedoch ebnet der weiteren Usurpation des juristischen Denkens durch das politische Streben den Weg.

Dass die Verwaltung selbst in ihren Entscheidungssituationen eine Perspektive einnimmt und einnehmen muss, die den juristischen Horizont überschreitet, ist selbstverständlich ${ }^{60}$ - aus ihrer Sicht bilden verwaltungsrechtliche Fragen einen von vielen Aspekten bei der Bewältigung eines Problems (der im Einzelfall hinderlich oder willkommen sein mag). Sobald sich jedoch die Rechtswissenschaft und das juristische Denken in der Rechtspraxis eine solche erweiterte Sichtweise aneignen wollen, leidet die Behandlung des Rechts der Verwaltung als Recht - und es leidet damit zugleich ein Kernbestandteil der Rechtsstaatlichkeit. Die Ausrichtung des verwaltungsjuristischen Denkens auf die Situation des richterlichen Entscheidens korrespondiert unmittelbar mit jenem außerordentlich hohen Grad an Verrechtlichung, den das Handeln der Verwaltung in Deutschland von Verfassungs wegen erfährt: mit der rechtlichen Bindung der Exekutive hat das grundgesetzliche Rechtsstaatsprinzip auch das juristisch-dogmatische Denken im Bereich der Verwaltung inthronisiert. ${ }^{61}$ Gerade hierin liegt die eigentliche Sicherung gegen die politische Beliebigkeit und den politischen Missbrauch bei der Handhabung von verwaltungsrechtlichen Normen.

\section{Die Beschränkung eines Imports sozialwissenschaftlicher Erkenntnisse und Theorien in das verwaltungsjuristische Denken}

Ein weiterer wichtiger Punkt, welchen die Neue Verwaltungsrechtswissenschaft insbesondere durch ihre Bezüge zur Steuerungstheorie und zur Governance-Forschung thematisiert (wenngleich auch diese Fragestellung nicht neu ist), betrifft das Verhältnis der juristischen Arbeit auf dem Gebiet der Verwaltung zur sozialwissenschaftlichen Forschung. ${ }^{62}$ Wann darf sie und wann muss sie sich die Erkenntnisse aneignen, zu denen die Sozialwissenschaften gelangen - und vor allem: inwiefern kann sie das überhaupt? Dass sich andere Wissenschaftszweige mit Verwaltungsrecht (und mit

59 Siehe hierfür statt vieler Appel, Steuerungswissenschaft (Fn. 4), S. 230 und M. Ruffert, Die Methodik der Verwaltungsrechtswissenschaft in anderen Ländern der Europäischen Union, in: Schmidt-Aßmann/Hoffmann-Riem (Hrsg.), Methoden der Verwaltungsrechtswissenschaft, Baden-Baden 2004, S. 165 (202).

60 Siehe beispielsweise Trute, Leitmotive (Fn. 23), S. 16.

61 Stolleis, Entwicklungsstufen (Fn. 19), $\$ 2$ Rn. 47. Siehe dazu auch F. Ossenbühl, Grundlagen des Verwaltungsrechts, Die Verwaltung 40 (2007), S. 125 (126).

62 Ausführlich dazu von Arnauld, Wissenschaft (Fn. 4), S. 65; J. Lüdemann, Rechtsetzung und Interdisziplinarität in der Verwaltungsrechtswissenschaft, in: Funke/Lüdemann (Hrsg.), Öffentliches Recht und Wissenschaftstheorie, Tübingen 2009, S. 119 (122ff.); Möllers, Methoden (Fn. 2), $\$ 3$ Rnrn. 47 ff.; ders., Theorie, Praxis und Interdisziplinarität in der Verwaltungsrechtswissenschaft, VerwArch 93 (2002), S. 22 (40) sowie T. Vesting, Nachbarwissenschaftlich informierte und reflektierte Verwaltungsrechtswissenschaft - „Verkehrsregeln“ und „Verkehrsströme“, in: Schmidt-Aßmann/Hoffmann-Riem (Hrsg.), Methoden der Verwaltungsrechtswissenschaft, Baden-Baden 2004, S. 253. 
dem Recht überhaupt) beschäftigen und beschäftigen müssen, erscheint selbstverständlich: Soziologen untersuchen das Recht als soziales Phänomen und als gesellschaftlichen Steuerungsfaktor; Politikwissenschaftler betrachten sich den Prozess des Zustandekommens rechtlicher Normen und die politischen Folgen, die durch Normen ausgelöst werden; Wirtschaftswissenschaftler analysieren das Recht unter dem Gesichtspunkt von Kosten und Nutzen; Psychologen befassen sich mit dem Problem, was Menschen dazu veranlasst, dem Gesetz entsprechend zu handeln oder es zu verletzen - und einige weitere Beispiele ließen sich finden. Bei ihrer Beschäftigung mit dem Recht betreibt keine dieser Wissenschaftsdisziplinen Rechtswissenschaft, und keine von ihnen behauptet auch nur, dies zu tun. Eine wissenschaftstheoretische Abgrenzung der Rechtswissenschaft gegenüber den Sozialwissenschaften bei der Erforschung des Erkenntnisgegenstands ,Recht' ist aus der Verschiedenheit der Erkenntnisziele heraus zu entwerfen. Den Nenner, auf den sie gebracht werden kann, markiert der Gesichtspunkt, dass nur die Rechtswissenschaft dazu anleiten soll, Rechtmäßigkeit und Rechtswidrigkeit zu unterscheiden. Andere Maßstäbe des Handelns (wie z.B. ökonomische Effizienz, ethische Vorbildlichkeit oder sozialpolitische Folgen) hat sie nur dort zu beachten, wo sich die rechtlichen Normen auf einen solchen Maßstab beziehen. Soziale Funktionen des Rechts sind für eine Jurisprudenz, welche sich auf die Unterscheidung von Rechtmäßigkeit und Rechtswidrigkeit konzentriert, allenfalls am Rand relevant. ${ }^{63}$ Demgegenüber befassen sich Steuerungswissenschaft und Governance-Forschung gerade nicht mit dem Recht in seiner Eigenschaft als eine autonom und absolut zu denkende Größe, sondern es wird das Recht auch hier als ein sozialer Faktor verstanden (nämlich als ein Steuerungsfaktor), so wie andere Wissenschaftszweige das Recht als ökonomischen oder politischen Faktor behandeln. Steuerungswissenschaft und Governance-Forschung sind somit von ihrem Erkenntnisziel her Sozialwissenschaften. ${ }^{64}$

Wenn es im folgenden darum geht, Schranken zu finden und zu untersuchen, innerhalb derer die Wissenschaft vom Recht der Verwaltung auf sozialwissenschaftliche Theorien zurückgreifen darf, sind verschiedene Konstellationen zu unterscheiden: ${ }^{65}$

1. Dass die Erkenntnisse anderer Wissenschaften hilfreich sein können, wenn es sich darum handelt, einen Sachverhalt zu erforschen ${ }^{66}$ um diesen juristisch prüfen zu

63 Ähnlich wohl Henke, Wandel (Fn. 58), S. 547. Siehe demgegenüber jedoch W. Hoffmann-Riem, Rechtswissenschaft als Rechtsanwendungswissenschaft, Lernzielthesen zur Integration von Rechtsund Sozialwissenschaft, in: Hoffmann-Riem (Hrsg.), Sozialwissenschaften im Studium des Rechts, Bd. II, München 1977, S. 1 (2) sowie Schmidt-Aßmann, Ordnungsidee (Fn. 7), S. 21.

64 Vgl. auch Meyer, Zweck (Fn. 7), S. 376.

65 Weil eine Differenzierung wie die folgende bei vielen Stellungnahmen zum Verhältnis zwischen den Sozialwissenschaften und der Wissenschaft vom Verwaltungsrecht fehlt, lässt sich die Reichweite entsprechender Aussagen über das Erfordernis eines interdisziplinären Austauschs häufig nur schwer ermessen. Soll man vermuten, dass dies mitunter gewollt ist?

66 Hierzu allgemein Schmidt-Aßmann, Methoden (Fn. 39), S. 407 f. 
können, steht außer Frage. Dies gilt in gleicher Weise für die Natur- und Ingenieurwissenschaften (so zum Beispiel, wenn es darum geht, technische Risiken zu ermitteln) wie für die Sozialwissenschaften (so zum Beispiel bei der Kalkulation der wirtschaftlichen und sozialen Auswirkungen von Großprojekten der Infrastruktur). ${ }^{67} \mathrm{Zu}$ bedenken ist dabei, dass es aus juristischer Sicht letztendlich immer die Rechtsnormen sind, die bestimmen, in welcher Hinsicht, in welchem Umfang und gegebenenfalls auch mit welchen Mitteln der fragliche Sachverhalt aufgeklärt werden soll; entsprechende Regelungen lassen sich sowohl im materiellen Recht ${ }^{68}$ als auch im Verfahrensrecht ${ }^{69}$ finden. Weil eine solche sachverhaltsbezogene Verarbeitung der Erkenntnisse anderer Wissenschaftszweige aus juristischer Sicht stets auf rechtliche Normen bezogen erscheint, kann der nichtjuristische Sachverstand nur eine dienende und untergeordnete Rolle einnehmen - dies gilt für die rechtsdogmatische Arbeit ebenso wie für die Tätigkeit der Justiz.

2. Schwieriger liegen die Dinge, wenn die Frage im Raum steht, inwiefern die Forschungsergebnisse der Sozialwissenschaften nützlich sein können, um den Inhalt einer rechtlichen Norm zu erfassen. Auch dies ist freilich kein neues Problem, dessen Entdeckung die Neue Verwaltungsrechtswissenschaft für sich beanspruchen könnte. Dass bestimmte Kenntnisse der Lebenswirklichkeit erforderlich sind, um überhaupt zu verstehen, in welche soziale Wirklichkeit der Gesetzgeber mit seiner Regelung eingreifen wollte, ist ein Gemeinplatz. ${ }^{70}$ „Wer die Lebenssachverhalte, auf die sich das Recht bezieht, weder kennt noch versteht, der kann auch den Inhalt der Rechtsvorschriften nicht verstehen, dem bleibt der Sinn der Rechtsvorschriften verborgen. “71 Zugespitzt ließe sich sagen, dass die gesamte juristische Gedankenwelt überhaupt erst durch dieses Wissen um das Tatsächliche ihre Bedeutung erhält. Der Wirklichkeitsausschnitt, auf den das Gesetz sich bezieht, wurde in der Literatur zum Teil unter dem Begriff eines "Normbereichs“ (Friedrich Müller/Ralph Christensen) ${ }^{72}$ und zum Teil unter dem Begriff eines „Realbereichs“ (Wolfgang Hoffmann-

67 Vgl. dazu auch E. Schmidt-Aßmann, Einige Überlegungen zum Thema: Die Wissenschaft vom Verwaltungsrecht, in: Die Wissenschaft vom Verwaltungsrecht (Die Verwaltung, Beiheft 2), Berlin 1999, S. 177 (180).

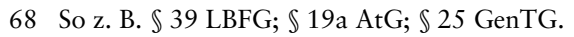

69 So z. B. $\mathbb{S} \mathbb{S} 24,26 \mathrm{VwVfG} ; \mathbb{1} 16$ AsylVfG; $\mathbb{S} \int 20 \mathrm{ff}$. SGB X.

70 Meyer, Zweck (Fn. 7), S. 352; Winkler, Verwaltungsrecht (Fn. 6), S. 15; vgl. auch Trute/Kühlers/Pilniok, Governance (Fn. 8), S. 184 f.

71 Winkler, Verwaltungsrecht (Fn. 6), S. 16. Rüthers (Methodenrealismus [Fn. 27], S. 58) formuliert als Grundsatz für alle Textwissenschaften: „Einen Text verstehen, das setzt zunächst voraus, die Frage und die Lage zu verstehen, auf die der Text eine Antwort sein sollte.“.

72 F. Müller/R. Christensen, Juristische Methodik, Bd. I, 10. Aufl., Berlin 2009, Rnrn. 235 ff.; kritisch zu diesem Begriff Hoffmann-Riem, Rechtsanwendungswissenschaft (Fn. 63), S. 6 f. (dort in Fn. 212). 
Riem $)^{73}$ thematisiert. ${ }^{74}$ Es spricht von vornherein nichts dagegen (und es ist sogar in gewissem Maß unerlässlich), dass sich Juristen die Erkenntnisse anderer Wissenschaftszweige aneignen, um diesen normrelevanten Ausschnitt der Realität zu erfassen. Trotz aller Schwierigkeiten und Differenzierungen, die hierbei in der Rechtspraxis bewältigt und von der Rechtswissenschaft reflektiert werden müssen, kann die prinzipielle Frage nach der Zulässigkeit und den Schranken dieses Rekurses auf die Erkenntnisse der Sozialwissenschaften klar beantwortet werden - es handelt sich letztlich um ein Problem, das jedem geläufig erscheint, der sich juristisch betätigt: soweit dem Juristen für das Erfassen der Realitäten sozialwissenschaftliche Ansätze nützlich erscheinen, darf er sich darauf stützen ${ }^{75}$ (für Erkenntnisse aus dem Bereich der Naturwissenschaften gilt auch in dieser Hinsicht dasselbe). Die prinzipielle Verteilung der Aufgaben zwischen Rechts- und Sozialwissenschaften, wie sie oben dargelegt worden ist, bleibt hierdurch unangetastet; aus juristischer Sicht kann den zuletzt Genannten wiederum nur eine dienende Rolle zukommen. ${ }^{76} \mathrm{Um}$ eine solche Definition und Eingrenzung dessen, was sozialwissenschaftliche Forschung für die verwaltungsrechtliche Arbeit bedeuten kann, wenn es um die Bestimmung des Norminhaltes geht (eine Begrenzung, die schlechterdings unerlässlich erscheint, solange es im Bereich der Verwaltung Recht geben soll, das man als solches erkennt), hat sich die Neue Verwaltungsrechtswissenschaft von vornherein nicht bemüht.

3. Die Fragen nach der Zulässigkeit und der Notwendigkeit eines Rückgriffs auf die Erkenntnisse der Sozialwissenschaften stellen sich dort am schwierigsten dar, wo es darum geht, bestimmte neu sich herausbildende Normenbestände als geltendes Recht zu identifizieren. ${ }^{77}$ Von den zuvor behandelten Fällen unterscheidet sich diese Konstellation insbesondere dadurch, dass nicht nur der Inhalt oder die Rechtsqualität eines einzelnen Satzes fraglich erscheint, sondern dass Unsicherheit darüber herrscht, ob man es bei einer bestimmten Art von Regelungen überhaupt mit rechtlichen Normen zu tun hat. Handelt es sich um rechtliche Normen, und wie begründet man deren juristische Geltung, wenn in einer transnationalen Arena gewisse Akteure, die man nach bisherigem Verständnis als Privatrechtssubjekte behandelt, gemeinsam mit anderen Akteuren, deren Maßnahmen man als hoheitlich einstuft, quasiverbindliche

73 W. Hoffmann-Riem, Methoden einer anwendungsorientierten Verwaltungsrechtswissenschaft, in: Schmidt-Aßmann/Hoffmann-Riem (Hrsg.), Methoden der Verwaltungsrechtswissenschaft, Baden-Baden 2004, S. 9 (36); ders., Juristische Verwaltungswissenschaft - multi-, trans- und interdisziplinär, in: Ziekow (Hrsg.), Verwaltungswissenschaften und Verwaltungswissenschaft, Berlin 2003, S. 45 (53); ebenso Voßkuble, Verwaltungsrechtswissenschaft (Fn. 7), $\mathbb{1} 1$ Rn. 29. Vgl. außerdem HoffmannRiem, Rechtsanwendungswissenschaft (Fn. 63), S. 6, wo vom „Sachbereich der Norm“ gesprochen wird.

74 Vgl. dazu auch Eifert, Verwaltungsrecht (Fn. 7), S. 301.

75 Siehe Voßkuble, Verwaltungsrechtswissenschaft (Fn. 7), $\$ 1$ Rn. 29 und Winkler, Verwaltungsrecht (Fn. 6), S. 34.

76 Vgl. dazu auch Winkler, Verwaltungsrecht (Fn. 6), S. 10 und 17.

77 Instruktiv hierzu Möllers, Wissenschaftstheorie (Fn. 4), S. $160 \mathrm{ff}$. 
Regelwerke erstellen ${ }^{78}$ (als Beispiel hierfür sei die Regulierung der Internetnutzung durch ICANN genannt $)^{79}$ ? In einem eng bemessenen Umfang sind vergleichbare Unsicherheiten jeder rechtlichen Ordnung bekannt - man denke an die Kontroverse über die Rechtsnatur von Verwaltungsvorschriften. ${ }^{80}$ Im Hinblick auf die neu entstehenden Steuerungsformen ist es richtig und unerlässlich zu untersuchen, wo und wie sich hierbei ein neues Konzept des Rechts herausbilden kann und woran man dieses erkennt. Es handelt sich dabei um Prozesse, die sich auch aus soziologischer Sicht beobachten und in Modellen abbilden lassen. Auf die entscheidende Frage nach der rechtlichen Geltung können soziologische Studien freilich keine Antworten liefern - diese originär juristische Frage nach der rechtlichen Geltung bedarf einer originär juristischen Antwort. Anknüpfen lässt sich dabei zunächst an Hans Kelsen, indem man sich auf die Regeln der Rechtserzeugung bezieht. ${ }^{81}$ Das Problem, wie eine Regelung als rechtliche Norm identifiziert werden kann, ist damit allerdings nicht gelöst: es wäre jetzt nämlich zu fragen, woran man die Regel der Rechtserzeugung als eine solche erkennt - dies insbesondere dann, wenn man es mit Rechtserzeugungsregeln zu tun hat, die dem traditionellen Rechtsetzungsmodus des Verfassungsstaates nicht mehr gänzlich entsprechen. ${ }^{82}$ Womit ist zu beginnen und wie geht man heran, wenn sowohl die Geltung bestimmter Sätze als rechtliche Normen als auch die entsprechenden Regeln über die Erzeugung des Rechts klärungsbedürftig erscheinen? Könnte es nicht sogar sein, dass gerade die Regeln über die Rechtserzeugung in bestimmten Konstellationen eines grundlegenden Wandels erst aus dem Bestand des erzeugten Rechts heraus erkannt werden können? Man gelangt an dieser Stelle zu den bereits weiter oben geäußerten Zweifeln zurück, ob die Rechtswissenschaft bei diesem Fragenkreis anders als methodenlos vorgehen kann. ${ }^{83} \mathrm{Ob}$ es sich so verhält, mag im hiesigen Kontext dahingestellt bleiben (dass ein methodisches Arbeiten unmöglich ist, lässt sich seinerseits schwer beweisen) - hier genügt es zu zeigen, wer dieses Problem zu bewältigen hat. Es sind dies wiederum nur die Juristen, indem sie die Rechtsqualität bestimmter Regelungen annehmen oder zurückweisen

78 Vgl. Trute, Leitmotive (Fn. 23), S. $28 \mathrm{ff}$.

79 Zur rechtlichen Einordnung des Handelns von ICANN ausführlich M. Weigele, Internet Corporation on Assigned Names and Numbers (ICANN), Staats-, europa- und völkerrechtliche Beurteilung, MMR 2013, S. 16.

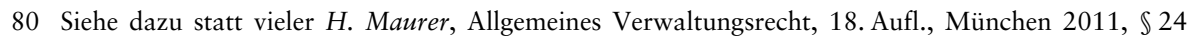
Rnrn. 2 ff.

81 Siehe dazu insbesondere H. Kelsen, Reine Rechtslehre, Studienausgabe, Tübingen 2008 (Nachdruck der 1. Aufl. von 1934), S. 74 ff.

82 Dass die Reine Rechtslehre ihren Wissenschaftlichkeitsanspruch nur unter den historischen Bedingungen der Positivität des Rechts erhebt (und man sollte hinzufügen: dass sie ihn überhaupt nur unter diesen Bedingungen zu erheben vermag), führt Möllers (Wissenschaftstheorie [Fn. 4], S. 161) zutreffend aus. Vgl. dazu auch bei Kelsen selbst: Rechtslehre (Fn. 81), S. 75.

83 Siehe dazu oben: B.II., sowie (in einem weiteren Zusammenhang) Haack, Staatstheorie (Fn. 34), S. $63 \mathrm{ff}$. 
müssen, wenn sie mit konkreten rechtlichen Fragen befasst sind ${ }^{84}$ Der Nutzen sozialwissenschaftlicher Forschungen für die juristische Arbeit reicht auch hier nicht über den Vorgang der Materialaufbereitung hinaus; die Entscheidung darüber, ob es sich bei den Normen eines bislang nicht bekannten Regelungstyps um Sätze des geltenden Rechts handeln muss, bildet ein Thema des juristischen Denkens. Inhalte, Quellen und Formen des Rechts können sich grundlegend ändern - schleichend oder abrupt; nicht zu ändern vermag sich indes der originäre Beruf des Juristen, dessen Eigenheit darin besteht, das geltende Recht eben als solches zu definieren, den Inhalt seiner Sätze aufzubereiten und die hieraus sich ergebenden Schlussfolgerungen zu ziehen, wenn es um seine Anwendung geht. Der Wissenschaft vom Recht der Verwaltung obliegt es, den Vertretern der verwaltungsrechtlichen Praxis zu zeigen, wie dies richtigerweise getan werden muss - auch diese Aufgabe, die freilich immer wieder neu zu bewältigen ist, bleibt als solche konstant. Dass die Verabschiedung von der Kategorie des Staates als juristischer Größe, wie sie in der Staats- und Verwaltungsrechtswissenschaft in einem wachsenden $\mathrm{Ma}$ zu beobachten ist, ${ }^{85}$ einer zukunftsweisenden Erörterung dieser Probleme keineswegs dienlich erscheint, ${ }^{86}$ sei an dieser Stelle nur kurz vermerkt. Auch wenn es zutrifft, dass die überkommenen Vorstellungen von der hoheitlichen Gewalt und vom Staat im Hinblick auf europäische und internationale Normerzeugungsstrukturen zurechtgerückt werden müssen, ${ }^{87}$ so würden doch Theorien, welche den Staat als juristische Kategorie für überholt erachten und aufgeben wollen, die Identifizierung des geltenden Rechts erschweren oder vereiteln, anstatt diese zu fördern.

Wenn wir gegenüber der Neuen Verwaltungsrechtswissenschaft dafür eintreten, dass sich die Wissenschaft vom Recht der Verwaltung noch wesentlich stärker als bisher auf ihr juristisches Kerngeschäft konzentriert, sind damit zwei Konsequenzen verbunden: es ist dies zum einen die entschiedene Selbstbeschränkung und Bescheidenheit der Verwaltungsrechtswissenschaft gegenüber allen Versuchen, andere Maßstäbe und Normen als das Recht zu behandeln; und es ist dies zum anderen ein offensiveres Engagement für ihre eigenen Ziele: die Unterscheidung von Rechtmäßigkeit

84 Wie dies praktisch abläuft, lässt sich beispielsweise beobachten, wo Rechtswissenschaft und Rechtspraxis die rechtsverbindlichen Sätze eines allgemeinen Verwaltungsrechts und eines Verwaltungsprozessrechts ausarbeiten, solange diese noch gar nicht oder nur zu einem kleinen Teil kodifiziert sind (was in Deutschland bis zum Inkrafttreten von VwVfG und VwGO der Fall gewesen ist und was sich im EU-Recht gegenwärtig als Aufgabe stellt); vgl. dazu von Arnauld, Wissenschaft (Fn. 4), S. 76 f.

85 Siehe statt vieler C. Möllers, Staat als Argument, 2. Aufl., München 2011, S. XLIX; vgl. auch Franzius, Governance (Fn. 8), S. $207 \mathrm{f}$.

86 Der Sache nach ebenso Spiecker genannt Döhmann, Verwaltungsrecht (Fn. 7), S. 1082 f. Ausführlich zur bleibenden Bedeutung des Staates für die Wissenschaft vom öffentlichen Recht Haack, L'État (Fn. 34), S. 107.

87 Einen eigenen Versuch hierzu haben wir anderenorts publiziert; siehe S. Haack, Der Staat mit den geteilten Organen, Paderborn u.a. 2013, S. 21 ff.; ders., Demokratie mit Zukunft? - Zwei Alternativen der Neukonzeption einer Staatsform, JZ 2012, S. 753 (756 ff.) - er läuft darauf hinaus, die supranationale Hoheitsgewalt als einen Bestandteil jedes einzelnen Mitgliedstaates zu deuten. 
und Rechtswidrigkeit (und damit verbunden: die Erkenntnis der rechtlichen Geltung), wobei die Erträge aus den Forschungen nichtjuristischer Wissenschaftsdisziplinen stets nur eine untergeordnete und vom Recht her bestimmte Bedeutung erlangen. Der hier vorgelegte Beitrag zu einer Läuterung der Verwaltungsrechtstheorie ist insofern auch als ein Plädoyer dafür zu lesen, die verkümmernde Disziplin der Verwaltungslehre zu fördern, ${ }^{88}$ um die Jurisprudenz im Bereich der Verwaltung gegen Anfragen zu schützen, für die sie nicht zuständig ist.

\section{Die Beschränkung einer auf die Praxis der Verwaltung fixierten Herangehensweise}

Bereits in dem vorangegangenen Abschnitt ${ }^{89}$ zur Trennung von rechtspolitischem und juristischem Denken wurde gezeigt, dass die Perspektive, welche die Verwaltung bei ihrer praktischen Tätigkeit einnimmt, zunächst einmal keine juristische ist. Ziel der behördlichen Arbeit ist die Bewältigung von sozialem, ökonomischem und ökologischem Konfliktstoff im weitesten Sinne. Dass die Verwaltung bei sämtlichen Maßnahmen ihre Bindung an Recht und Gesetz gemäß Art. 20 Abs. 3 GG im Blick haben muss, ändert hieran grundsätzlich nichts; Rechtsnormen bilden insofern nur einen von vielen Entscheidungsmaßstäben, dessen Gewicht bei der Vorbereitung und der Ausführung einer Verwaltungsmaßnahme je nach Zusammenhang variiert. Als relevant erweist er sich immer nur dann, wenn er eine bestimmte Vorgehensweise, welche die Behörde prinzipiell in Betracht ziehen könnte, aufgrund ihrer Unvereinbarkeit mit dem Rechtsinhalt ausschließt. ${ }^{90}$

Als komplizierter gestaltet sich das Problem, inwiefern die Bedürfnisse der Verwaltung bei ihrer praktischen Arbeit ausschlaggebend für das Verständnis verwaltungsrechtlicher Normen sein können. Immer wieder begegnet man in der Literatur zu den Einzelheiten des Besonderen Verwaltungsrechts (die nicht selten aus der Feder eines Rechtspraktikers stammt) der Überzeugung, dass eine solche Erwägung methodisch ohne weiteres zulässig sei - ohne dass diese Autoren eine solche Annahme begründen oder auch nur ausdrücklich äußern. Gerade ein solches Nachgeben gegenüber den scheinbaren oder wirklichen Erfordernissen der behördlichen Praxis bei der Anwendung rechtlicher Normen trägt jedoch zur Beliebigkeit bei, ${ }^{91}$ die am Ende als ein Gegenteil jener verrechtlichten Ordnung erscheint, welche vom Rechtsstaatsgrundsatz verlangt wird: das Prinzip der Rechtsstaatlichkeit impliziert, dass auch im Be-

88 Vgl. auch Kahl, Verwaltungsrecht (Fn. 10), S. 494 f.; Pauly, Verwaltungsrecht (Fn. 23), $\mathbb{S} 58$ Rn. 32 und Winkler, Verwaltungsrecht (Fn. 6), S. 27.

89 Siehe vorstehend: I.

90 Vosgerau (Theorie [Fn. 3], S. 211) spricht einprägsam davon, dass die Rechtswissenschaft den Entscheidungsträgern mitzuteilen habe, ,was sie nicht entscheiden können, weil es - u.a. durch das Verfassungsrecht - bereits entschieden ist" (Hervorhebung im Original).

91 Vgl. dazu auch M. Stolleis, Verwaltungsrechtswissenschaft in der Bundesrepublik Deutschland, in: Simon (Hrsg.), Rechtswissenschaft in der Bonner Republik, Frankfurt a. M. 1994, S. 227 (257). 
reich der Verwaltung vom Recht her (anstatt vom Zweck her) ${ }^{92}$ gedacht wird, wenn es um die behördliche Rechtsbindung geht. Dies wiederum heißt, dass auch die verwaltungsrechtlichen Normen den autonomen Gesetzmäßigkeiten und Eigenschaften des Rechts unterliegen, anhand derer sie interpretiert werden müssen..$^{93}$ Eine ausufernde und in sich nicht stimmige Kasuistik, wie man sie im Verwaltungsrecht immer häufiger findet, ${ }^{94}$ zeigt deutlich, dass es die rechtliche Regelung selbst ist, welche sich ohne eine feste, theoretisch fundierte Dogmatik in der Beliebigkeit auflösen wird. Aus rechtsstaatlicher Sicht erweist sich dies zunächst als ein Missstand im Hinblick auf das Gebot der Rechtssicherheit; ${ }^{95}$ vor allem jedoch verliert die verwaltungsrechtliche Norm hierdurch früher oder später ihren Charakter als Rechtssatz und endet als Regel der Zweckmäßigkeit. ${ }^{96}$

\section{Die Beschränkung von Reflexionen über die historische Bedingtheit des Rechts}

Insbesondere dann, wenn in der rechtswissenschaftlichen Literatur über solch abstrakte Kategorien wie den Verwaltungsakt nachgedacht wird, spielen Überlegungen zur historischen Bedingtheit des Rechts immer wieder eine besondere Rolle. ${ }^{97}$ Dass historischen Argumenten bei der juristischen Arbeit ein bedeutender Platz eingeräumt werden muss, ist freilich nicht zu bestreiten; diesen Platz finden sie im Rahmen des klassischen Auslegungskanons in Gestalt der genetischen und historischen Auslegungsmethode. ${ }^{98}$ Auch bei der Ermittlung von gewohnheitsrechtlichen Normen kommt es auf historische Einsichten an - für das Gebiet des Verwaltungsrechts sei nur auf die gewohnheitsrechtlich geltenden Rechtsinstitute des öffentlich-rechtlichen Ersatzleistungsrechts hingewiesen. Eine juristische Bedeutung, die darüber hinausreicht, besitzen Erkenntnisse über die Entwicklungsgeschichte des Verwaltungsrechts

92 Zum „Siegeszug des Zwecks“ in der Verwaltungsrechtsdogmatik S. Magen, Entscheidungen unter begrenzter Rationalität als Proprium des öffentlichen Rechts, in: Engel/Schön (Hrsg.), Das Proprium der Rechtswissenschaft, Tübingen 2007, S. 303 (304 f.).

93 In dieselbe Richtung Püttner, Entwicklungstendenzen (Fn. 20), S. 123. Dem Grunde nach wird dies auch von manchen Vertretern der Neuen Verwaltungsrechtswissenschaft anerkannt, vgl. Voßkuble, Verwaltungsrechtswissenschaft (Fn. 7), $\mathbb{1} 1 \mathrm{Rn} .28$.

94 Als Beispiele seien genannt: die Frage nach dem drittschützenden Charakter von Rechtsvorschriften im Baurecht (siehe dazu J. Hellermann, in: Dietlein/Burgi/Hellermann, Öffentliches Recht in Nordrhein-Westfalen, 4. Aufl., München 2011, Rnrn. 313 ff.), die Rechtsprechung zur „unmittelbaren Auswirkung" bei enteignungsgleichem und enteignendem Eingriff (dazu F. Ossenbühl/M. Cornils, Staatshaftungsrecht, 6. Aufl., München 2013, S. 299 ff., 343 f.) und die Abgrenzung von zivilrechtlichen und öffentlich-rechtlichen Streitigkeiten gemäß $\ 40$ Abs. 1 VwGO bei tatsächlichem Handeln einer Behörde (dazu S. Haack, in: Gärditz [Hrsg.], VwGO, Köln 2013, \40 Rnrn. 96 ff.).

95 Allgemein dazu C. Degenhart, Staatsrecht I, Staatsorganisationsrecht, 28. Aufl., Heidelberg 2012, Rnrn. $353 \mathrm{ff}$.

96 Aufschlussreich und bezeichnend erscheint es, dass die Anhänger einer governance-orientierten Verwaltungsrechtswissenschaft das Gesetz als eine „Orientierungsmarke in Regelungsstrukturen“ betrachten (so Franzius, Governance [Fn. 8], S. 217).

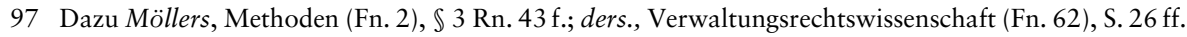
sowie Schulze-Fielitz, Qualität (Fn. 39), S. 53.

98 Zur Unterscheidung und Abgrenzung von genetischer und historischer Interpretation Müller/Christensen, Methodik (Fn. 72), Rnrn. 360 f. 
nicht. Zur Frage der Stichhaltigkeit einer rechtswissenschaftlichen Lehre tragen Beobachtungen nichts bei, die diesen in Frage stehenden Ansatz aus den Interessen von bestimmten politischen Kräften in einer konkreten historischen Situation heraus deuten ${ }^{99}$ - Beobachtungen zum Beispiel, wonach der staatsrechtliche Positivismus eines Paul Laband auf jene politischen Interessen zurückgeführt werden müsse, die sich innerhalb des Deutschen Reiches von 1871 als herrschend erwiesen. ${ }^{100}$ Ähnliches gilt für die Ansicht, welche das klassische juristische Denken im Verwaltungsrecht dem „theoretischen Rationalismus“ und dem „politischen Liberalismus“ zuordnet. ${ }^{101}$ Könnte es sich darum handeln, dass man die bleibende Gültigkeit von juristischen Einsichten einer bestimmten Epoche bestreitet (oder dass man sogar bestreitet, nach entsprechenden Einsichten überhaupt nur suchen zu dürfen), ${ }^{102}$ um auf diese Weise jede Art von epochenübergreifender juristischer Logik kleinzureden, wenn nicht sogar gänzlich zu diskreditieren? Was damit bezweckt wird, liegt auf der Hand: man steigert auf diese Weise ein weiteres Mal die politische Verfügbarkeit jedes juristischen Denkens. Die Ablehnung eines epochenübergreifenden juristischen Erkenntnisgewinns und die Betonung der historischen Bedingtheit des Rechts suggerieren, dass die Wissenschaft vom öffentlichen Recht (ebenso wie dieses selbst) keine eigene Dignität hat und deshalb dem politischen Willen gefügig gemacht werden darf. Hinzu kommt nicht selten die Absicht, durch Hinweise auf die historischen Bedingungen der juristischen Einsicht jene (bislang gültigen) Theoreme zu relativieren, die dem Rechtsinterpreten als hinderlich und überwindungsbedürftig erscheinen. Vermeiden lässt sich dies insbesondere durch die Entwicklung einer Dogmatik, die Begrifflichkeiten und Kategorien kennt, welche (trotz aller Änderungen in den Details) über den Epochenwechsel hinaus konstant bleiben müssen ${ }^{103}$ - als Beispiele für solche grundlegenden Bausteine des juristischen Denkens sind Anspruch, Vertrag oder Rechtskraft zu nennen.

Die hier formulierte Empfehlung, Reflexionen über die historische Bedingtheit des Rechts im Rahmen der juristischen Argumentation zu beschränken, lässt sich auch auf eine abstraktere, in der Auseinandersetzung mit der Wiener rechtstheoretischen

99 Ähnlich F. Hase, Das Verwaltungsrechtsverhältnis, Überlegungen zu einem Grundbegriff des Öffentlichen Rechts, Die Verwaltung 38 (2005), S. 453 (459); anders jedoch Henke, Wandel (Fn. 58), S. 541 und Möllers, Methoden (Fn. 2), $\mathbb{} 3$ Rn. 44.

100 So z. B. Möllers, Wissenschaftstheorie (Fn. 4), S. 171.

101 So z. B. Pöcker, Kommunikation (Fn. 12), S. 510; in dieselbe Richtung Park, Rechtsfindung (Fn. 11), S. 292 („liberalistische Einseitigkeit“).

102 Vgl. demgegenüber Hase, Verwaltungsrechtsverhältnis (Fn. 99), S. 459 und Winkler, Verwaltungsrecht (Fn. 6), S. 11; siehe hierzu ferner Bumke, Methodik (Fn. 6), S. 119 f.

103 Dazu auch S. Haack, Ordnungstranszendierende Verfassungsdogmatik im Kontext der Staatlichkeitsfrage, Anmerkungen zum Lissabon-Urteil des Bundesverfassungsgerichts aus staats- und rechtstheoretischer Sicht, Rechtstheorie 43 (2012), S. 325 ff. 
Schule zu entwickelnde Weise rechtfertigen: ${ }^{104}$ aufgrund des Anspruchs jedweder rechtlichen Ordnung, als unverbrüchlich zu gelten, ${ }^{105}$ wird die Rechtswissenschaft bereits durch die Besonderheiten ihres Erkenntnisgegenstandes zu einer ahistorischen Betrachtungsweise gezwungen. Zwar kennt das juristische Denken die Entstehungsgeschichte der Norm und den Bestand der vorherigen Regelungen als Auslegungsmittel - indem es sich jedoch auf das Entweder-Oder von Rechtmäßigkeit und Rechtswidrigkeit richtet, bleibt ihm die Idee der Geschichtlichkeit fremd. Zwar kann die Geltung von rechtlichen Normen durch diese selbst oder durch andere Normen in zeitlicher Hinsicht begrenzt sein - der juristische Geltungsanspruch als solcher ist nichtsdestoweniger zeitblind. Aus juristischer Sicht ist die Zeit nichts anderes als ein Tatbestandsmerkmal der Norm, das deren Anwendungsbereich definiert. ${ }^{106}$ Dass eine rechtliche Norm außer Kraft treten kann, ist über diese Frage der Eröffnung ihres Tatbestandes hinaus juristisch nicht relevant. Dies jedoch macht es unmöglich, in ein und derselben Angelegenheit zugleich geschichtlich und rechtlich zu argumentieren. Genauso wie man sich dem Recht der Verwaltung entweder als Jurist oder als Sozialwissenschaftler, nicht jedoch in diesen beiden Rollen auf einmal zuwenden kann, ist es nicht möglich, zugleich als Historiker und als Jurist damit beschäftigt zu sein. ${ }^{107}$ Dies hat auch die Theorie des Verwaltungsrechts zu beachten.

\section{Die Situation des juristischen Konflikts und der Klage als Dreh- und Angelpunkt der Verwaltungsrechtstheorie}

Eine Theorie des Verwaltungsrechts, die sich in der geschilderten Weise von disziplinfremden, unwissenschaftlichen und unjuristischen Perspektiven verabschiedet hat, kann sich wesentlich stärker als bisher auf ihr eigentliches Erkenntnisziel: die Unterscheidung von Rechtmäßigkeit und Rechtswidrigkeit, ${ }^{108}$ konzentrieren. Um so dringender zu beantworten bleibt an dieser Stelle die Frage, wo ein solchermaßen aufgeklärtes und geläutertes verwaltungsrechtliches Denken nach dem Ausscheiden

104 Siehe dazu insbesondere Kelsen, Rechtslehre (Fn. 81), S. 21 f.; von Interesse bleiben hierzu auch verschiedene Schriften von Adolf Julius Merkl, siehe dens., in: Klecatsky/Marcic/Schambeck (Hrsg.), Die Wiener rechtstheoretische Schule, Bd. 1, Neuauflage 2010, S. 883 ff. (913 ff.).

105 Haack, Verfassungsdogmatik (Fn. 103), S. 341 ff. Ausnahmen hiervon sind im Kontext historischer Umbrüche denkbar, wenn eine provisorische rechtliche Ordnung in Kraft gesetzt wird, um einen friedlichen Ablauf der Verfassunggebung zu sichern; man könnte insofern von „Interimsordnungen“ sprechen.

106 Vgl. Kelsen, Rechtslehre (Fn. 81), S. 22.

107 Dies ist freilich rein begrifflich gemeint - dass es Wissenschaftler gibt, die beide Berufe hervorragend ausfüllen, steht außer Frage. - Den Widerspruch, der hierbei auftreten muss, beschreibt M. Stolleis wie folgt: „Der Öffentlichrechtler, der sich in Lehre und Forschung mit den dogmatischen Grundfiguren des Verwaltungsrechts beschäftigt und zugleich als Rechtshistoriker wissenschaftsgeschichtliche Studien zur Entstehung des Verwaltungsrechts betreibt, gerät in einen eigentümlichen Zwiespalt. Im geltenden Recht vertritt er Thesen zum Allgemeinen Teil des Verwaltungsrechts, für deren relative ,Richtigkeit' innerhalb des ,Sprachspiels Rechtswissenschaft' er eintritt, deren historische Bedingtheit er aber als Rechtshistoriker nicht verkennen kann" (Entwicklungslinien der verwaltungsrechtlichen Dogmatik im industriellen Zeitalter, BWV 1990, S. 152).

108 Siehe oben: B.I. 
aller dieser Gesichtspunkte ansetzen soll - das zu untersuchen, ist das finale Anliegen des hier vorgelegten Essays. Mit anderen Worten: es soll darum gehen, ein belastbares theoretisches Fundament für die etablierten juristischen Arbeitsmethoden im Verwaltungsrecht zu gewinnen. Zurückzugreifen ist hierfür zunächst auf jene (zuvor bereits erwähnten ${ }^{109}$ Kategorien, welche unabhängig von der konkreten rechtlichen Ordnung als gültig erscheinen, indem sie sich überall dort, wo Recht existiert, in der einen oder anderen Ausprägung nachweisen lassen - als einige einfache Beispiele seien aus dem materiellen Recht die Kategorien ,Gebot' und ,Verbot' oder aus dem Prozessrecht die Kategorien ,Klage' und ,Rechtskraft' genannt. ${ }^{110}$ Es gehört zu den klassischen Aufgaben der Jurisprudenz im Verwaltungsrecht (und darüber hinaus) zu erfassen, wie die konkrete rechtliche Ordnung, die auf den Ordnungsvorstellungen der politisch geeinten Gemeinschaft beruht, ${ }^{111}$ diese Grundkategorien des juristischen Denkens gewendet und ins Werk gesetzt hat. Dogmatische Studien zum Inhalt des Rechts finden hier ihren Platz. Weniger gründlich durchdrungen wurde bislang das Problem, welchen grundlegenden Zusammenhang das juristische Denken in den Blick nehmen muss, um diese Aufgabe auf die bestmögliche Art zu erfüllen. Die hergebrachte Dogmatik des Verwaltungsrechts, wie sie insbesondere durch Otto Mayer auf den Weg gebracht worden war, ${ }^{112}$ hatte als einen solchen Dreh- und Angelpunkt ihres Denkens den behördlichen Rechtsakt, insbesondere in der Gestalt des Verwaltungsaktes, behandelt. ${ }^{113}$ In deutlicher Abgrenzung hierzu versuchte man seit den siebziger Jahren des vorigen Jahrhunderts, den Begriff ,Rechtsverhältnis' zum

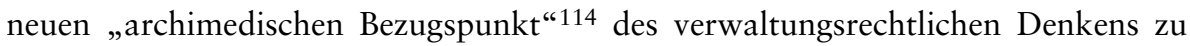
machen. ${ }^{115}$ Von den Kritikern dieser Lehre wurden indes schon recht bald die in-

109 Siehe oben: C.IV.

110 Vgl. dazu auch Winkler, Verwaltungsrecht (Fn. 6), S. 18.

111 Näher hierzu Haack, Staatstheorie (Fn. 34), S. 72 ff.

112 Siehe hierzu statt vieler Stolleis, Entwicklungsstufen (Fn. 19), $\$ 2$ Rnrn. $60 \mathrm{ff}$.

113 Vgl. Appel, Steuerungswissenschaft (Fn. 4), S. 252; Eifert, Verwaltungsrecht (Fn. 7), S. 290 und Pauly, Verwaltungsrecht (Fn. 23), $\mathbb{} 58$ Rn. 18; siehe dazu ferner auch Bauer, Verwaltungsrechtslehre (Fn. 19), S. 308 ff. und J. Pietzcker, Das Verwaltungsrechtsverhältnis - Archimedischer Punkt oder Münchhausens Zopf?, Die Verwaltung 30 (1997), S. 281 (297f.). Wenn in der Literatur zum Teil von einer „Handlungsformendogmatik“ die Rede ist (so R. Gröschner, Vom Nutzen des Verwaltungsrechtsverhältnisses, Die Verwaltung 30 [1997], S. 301 [319]), bleibt der Unterschied (jedenfalls in unserem Kontext) gering: im Zentrum der dogmatischen Konstruktion steht in jedem Fall die Rechtsnatur der behördlichen Handlung.

114 So Häberle, Pluralismus (Fn. 57), S. 250, ähnlich auch auf S. 256.

115 Herauszuheben sind u.a. N. Achterberg, Rechtsverhältnisse als Strukturelemente der Rechtsordnung, Prolegomena zu einer Rechtsverhältnistheorie, Rechtstheorie 9 (1978), S. 385; ders., Die Rechtsordnung als Rechtsverhältnisordnung, Berlin 1982, passim; Gröschner, Nutzen (Fn. 113), S. 301; Hase, Verwaltungsrechtsverhältnis (Fn. 99), S. 453; Henke, Wandel (Fn. 58), S. 542 f. sowie E. Vosniakou, Beiträge zur Rechtsverhältnistheorie, 1992, passim. Zur Vorgeschichte der Begrifflichkeit ,Rechtsverhältnis‘ siehe Bauer, Verwaltungsrechtslehre (Fn. 19), S. 316. 
haltliche Armut und die Konturenlosigkeit der Kategorie ,Rechtsverhältnis' bemerkt. ${ }^{116}$ Sachlich erscheint dieser Einwand berechtigt: wenn überhaupt, konnte die Figur des Rechtsverhältnisses wohl nur bei dauerhaften Beziehungen zwischen der hoheitlichen Gewalt und dem Bürger etwas zur rechtlichen Klärung beitragen (man dachte dabei insbesondere an das Recht der Leistungsverwaltung) ${ }^{117}$ - dies zum Beispiel im Hinblick auf Nebenpflichten, die man hieraus herleiten wollte. ${ }^{118}$

Dass die Lehre vom Rechtsverhältnis jene Erwartungen nicht erfüllt hat, die von ihr selbst geweckt worden waren, ${ }^{119}$ schließt es freilich nicht aus (und lässt es auch nicht als überflüssig erscheinen), erneut nach einem solchen Dreh- und Angelpunkt des verwaltungsrechtlichen Denkens zu suchen. Auch ein bloßes Beharren auf der bisherigen rechtsaktorientierten Dogmatik genügt indes nicht, um das Verwaltungsrecht in der gegenwärtigen Phase des Wandels fest genug zu fundieren: deren Schwachstellen, die sowohl seitens der Rechtsverhältnislehre als auch seitens der Neuen Verwaltungsrechtswissenschaft oft genug festgestellt worden sind, ${ }^{120}$ müssen hier nicht noch einmal aufgezeigt werden. Entscheidend für den hiesigen Kontext erscheint es vor allem, dass die herkömmliche Lehre durch ihre Ausrichtung auf die Handlungsform des Verwaltungsakts ihr eigenes Leistungsvermögen zur rechtsdogmatischen Systembildung schwächt. Zwar bildet sie, indem sie zwischen Verwaltungsakten und sonstigen Maßnahmen differenziert, die rechtliche Überordnung der hoheitlichen Gewalt und somit das Wesenskennzeichen der modernen Staatlichkeit ab. Es ist jedoch keineswegs so, dass es bei dem juristischen Umgang mit diesem Recht auf jene Überordnung immer und überall ankommt: sehr oft wird gerade dieser Gesichtspunkt als unproblematisch erscheinen und keiner juristischen Klärung bedürfen. Andere wesentliche Zusammenhänge vermag die Konzentration auf die

116 Siehe hierfür beispielsweise H. Maurer, Diskussionsbeitrag, VVDStRL 45 (1987), S. 280; H. Meyer, Diskussionsbeitrag, VVDStRL 45 (1987), S. 272 (,das inhaltsloseste Rechtsinstrument, das je angeboten worden ist“) und Pietzcker, Verwaltungsrechtsverhältnis (Fn. 113), S. 284; zurückhaltend auch W. Löwer, Rechtsverhältnisse in der Leistungsverwaltung, NVwZ 1986, S. 793 (794).

117 Siehe dazu H. Hill, Rechtsverhältnisse in der Leistungsverwaltung, NJW 1986, S. 2602 (2607ff.); P. Krause, Rechtsverhältnisse in der Leistungsverwaltung, VVDStRL 45 (1987), S. 212, (224ff.); Löwer, Rechtsverhältnisse (Fn. 116), S. 795 ff. und F. E. Schnapp, Rechtsverhältnisse in der Leistungsverwaltung, DÖV 1986, S. 811 (814 ff.).

118 So z. B. Hase, Verwaltungsrechtsverhältnis (Fn. 99), S. 463.

119 Beispielhaft hierfür Achterberg, Rechtsverhältnisordnung (Fn. 115), S. 73 (der die Rechtsverhältnislehre in eine Reihe mit der Reinen Rechtslehre gestellt hat) und Häberle, Pluralismus (Fn. 57), S. $251 \mathrm{ff}$.

120 Im Unbehagen gegenüber den Schwächen der herkömmlichen rechtsaktorientierten Dogmatik treffen sich somit die Rechtsverhältnislehre und die Neue Verwaltungsrechtswissenschaft, die im übrigen eher in einem Gegensatz zueinander gesehen werden (vgl. J. Ipsen, Neue Entwicklungen im Allgemeinen Verwaltungsrecht, Rechtsverhältnislehre versus Steuerungswissenschaft?, Ad Legendum 2011, S. 100); beide wollten - auf jeweils eigene Weise - die rechtsaktbezogene Herangehensweise ergänzen. Siehe dazu statt vieler P. Häberle, Diskussionsbeitrag, VVDStRL 45 (1987), S. 251 (252) (für die Lehre vom Rechtsverhältnis) sowie Appel, Steuerungswissenschaft (Fn. 4), S. 276 (für die Neue Verwaltungsrechtswissenschaft). 
Handlungsform und den Rechtsakt indes nicht sichtbar zu machen. ${ }^{121}$ Man könnte aus diesem Grund sagen, dass die hergebrachte verwaltungsrechtliche Lehre ein tragfähiges Fundament deshalb nicht herzustellen vermochte, weil sie durch ihre Fixierung auf die Handlungsform des Verwaltungsakts den eigentlichen Schwerpunkt des dogmatischen Theoriengebäudes verkannt hat; demgegenüber konnte die Lehre vom Rechtsverhältnis ein solches tragfähiges Fundament nicht begründen, weil der begriffliche Untergrund, den sie hierfür gewählt hat (es war dies der Untergrund in der Gestalt des Begriffes ,Rechtsverhältnis‘) zu weich war. Während die hergebrachte Dogmatik heutzutage als schiefer Turm vor uns steht, begegnet uns die Rechtsverhältnislehre als Bauruine. Auch die Neue Verwaltungsrechtswissenschaft lässt sich mit diesen Metaphern beschreiben: über der Ambition, das Theoriengebäude der Verwaltungsrechtswissenschaft in einer völlig neuen Dimension zu konzipieren, ist es bei kühnen Entwürfen geblieben; ein Fundament, welches sich für die juristische Arbeit als brauchbar erweist, liegt ihr bis jetzt nicht zugrunde.

An dieser Stelle schlagen wir nunmehr vor, die Situation eines juristischen Konflikts - und damit letztendlich: die Situation einer Klage - als den gesuchten Dreh- und Angelpunkt der Verwaltungsrechtswissenschaft zu begreifen. ${ }^{122}$ Die These, die hiermit aufgestellt wird, soll daher lauten, dass die Unterscheidung von Rechtmäßigkeit und Rechtswidrigkeit immer nur in einer solchen Konfliktsituation zu gewinnen sein kann. Es stimmt zwar (und es stellt nicht mehr dar als eine Binsenweisheit), dass sich die rechtliche Ordnung in den meisten Situationen verwirklicht, ohne dass ein Jurist dabei nachhilft - ein Großteil der Rechtsnormen wird seitens der Rechtsadressaten befolgt. Aus soziologischer Sicht bildet dies die normale Funktionsweise des Rechts. ${ }^{123}$ Im Irrtum befindet sich allerdings, wer davon ausgeht, dass eine solche soziologische Beobachtung der normalen Funktionsweise von rechtlichen Normen einen geeigneten Anknüpfungspunkt zur Verankerung der juristischen Dogmatik darstellen kann. Ein Denken, das darauf abzielt, anhand der Kriterien des Rechts über Rechtmäßigkeit und Rechtswidrigkeit zu befinden, geht seiner Idee nach immer von der Situation des Konflikts aus; juristisch zu denken heißt: zu denken in einer speziellen, auf den rechtlichen Maßstab bezogenen Entscheidungssituation. Unabhängig davon, ob diese in der Wirklichkeit auftritt oder lediglich in den Gedanken durchgespielt wird, handelt es sich um jene Situation, in der sich das Recht als solches bewährt und beweist; die Funktion der Dogmatik liegt darin, das Recht für solche

121 Kritisch bereits H. Faber, Auf einem dritten Weg, in: FS Ridder, Neuwied 1989, S. 291 (292 ff.).

122 Als anregend für ein solches Konzept haben sich u. a. erwiesen: G. Burdeau, Traité de science politique, Bd. I, Paris 1949, Rn. 139 und Winkler, Verwaltungsrecht (Fn. 6), S. 13.

123 Anschaulich beschrieben hat dies bereits E. Ehrlich, Grundlegung der Soziologie des Rechts, München 1913 (Neudruck 1929), S. 50 ff. 
Konfliktsituationen zuzubereiten. ${ }^{124}$ Erst dadurch, dass die Dogmatik das objektive Recht aus der Perspektive der juristischen Ur-Situation, sprich also: vom Prozess her erfasst, lässt sie dieses handhabbar werden. ${ }^{125}$ Rechtliche Normen beantworten im Grunde nur eine einzige Frage: es ist dies die Frage danach, wer vor dem Richter obsiegt. ${ }^{126}$ Welche Antwort sie hierauf geben, lässt sich immer nur im Hinblick auf ein konkretes Geschehen bestimmen; es gibt kein Recht ohne Situation. Carl Schmitts mehrdeutiges und merkwürdiges Diktum, alles Recht sei Situationsrecht, ${ }^{127}$ enthält von dieser Warte her gesehen einen fassbaren Sinn: es besagt, dass rechtliche Normen nur im Hinblick auf die konkrete Konfliktsituation gedacht und erkannt werden können. Um diesen Gesichtspunkt so unmissverständlich wie möglich zu präsentieren, sei mit anderen Worten gesagt: nur im Hinblick auf einen Subsumtionsvorgang lassen sich Sätze als Normen verstehen. Betont sei zudem ein weiteres Mal, dass es für diese Prämisse eines jeden juristischen Denkens auf die Realität dieses Konfliktes nicht ankommt; maßgeblich ist allein der Gesichtspunkt, dass mit dem Norminhalt der Konflikt von vornherein mitgedacht werden muss. Nichts anderes kennzeichnet letztendlich das Tätigsein der Verwaltung, zu deren Angelegenheiten es zählt, das faktische Ausbrechen eines Konflikts zu verhindern. Ein solches verwaltungsrechtliches Denken vom Rechtskonflikt her ließe die Rechtsverhältnislehre sogar dort als entbehrlich erscheinen, wo es um den Umstand der Dauerhaftigkeit von Rechtsverhältnissen geht (wie er uns insbesondere im Bereich der Leistungsverwaltung begegnet): aus juristischer Sicht ist auch dieser Umstand der Dauerhaftigkeit nur im Hinblick auf den (praktisch zu vermeidenden) Rechtskonflikt relevant - dies insbesondere dann, wenn man Ansprüche aus der Verletzung von Nebenpflichten behauptet oder aus Verstößen gegen den Grundsatz des Vertrauensschutzes Abwehrrechte herleitet.

124 Hierum geht es letztlich auch dort, wo man von der „Anwendungsbezogenheit“ der Rechtswissenschaft spricht, vgl. Waldhoff, Rechtsdogmatik (Fn. 27), S. 31. Was sollte nämlich die „Anwendung“ einer Rechtsnorm anderes sein als die Beurteilung eines Sachverhaltes anhand dieser Norm im konkreten Konflikt - unabhängig davon, ob dieser sich in der Theorie oder in der Wirklichkeit findet? Vgl. dazu auch Möllers, Verwaltungsrechtswissenschaft (Fn. 62), S. 56 f.

125 In die richtige Richtung zielt deshalb die Formulierung, es seien „die Arbeit mit Fällen und der Zwang, Entscheidungen zu treffen, [...] das größte methodische Privileg der Rechtswissenschaften “ (so Möllers, Verwaltungsrechtswissenschaft [Fn. 62], S. 56); besser wäre es jedoch, noch etwas weiter zu gehen: ein juristisches Denken ohne (imaginäre oder geschehene) Fälle ist von vornherein unvorstellbar. Vgl. ferner auch Breuer, Verwaltungsrechtswissenschaft (Fn. 14), S. 286, der die unmittelbare Beziehung eines solchen juristischen Denkens zu dem Grad der Verrechtlichung im Bereich der Verwaltung und somit letztlich zu dem Rechtsstaatsgrundsatz andeutet.

126 Den Prozess als die „Anwendungsebene“ einer „Normebene“ gegenüberzustellen (so Pöcker, Kommunikation [Fn. 12], S. 528), wird dem Problem aus diesem Grund nicht gerecht.

127 C. Schmitt, Politische Theologie, 7. Aufl., Berlin 1996, S. 19. Interessant erscheint aus hiesiger Sicht auch die Erwiderung H. Hellers (Staatslehre, 3. Aufl., Tübingen 1963, S. 255), der dies bestreitet, indem er auf die stabilisierende Funktion der Norm (in diesem Fall der Verfassung) aufmerksam macht - hierdurch jedoch soziologisch argumentiert. Obgleich C. Schmitt bei seinem Diktum auf andere Zusammenhänge gezielt haben mag, kam er dem Wesen des rechtlichen Denkens dabei näher als $H$. Heller, bei dem sich auch hier soziologisches und juristisches Denken vermischen. 
Gerade eine solche Konzentration des juristischen Denkens auf die Situation von Konflikt und richterlicher Entscheidung stellt eine passende Antwort dar auf die wachsende Komplexität der rechtlichen Ordnung, ${ }^{128}$ weil sie den Dogmatiker dazu zwingt und ihn zugleich darauf festlegt, für jeden denkbaren Anspruch zu untersuchen, was aus juristischer Sicht (unmittelbar oder mittelbar) relevant ist. Durch eine solche Herangehensweise ließe sich die verwaltungsrechtliche Dogmatik auch in jenen zentralen Fragen fundieren, die (nicht ohne Grund) bislang ungelöst sind: ${ }^{129}$ zu denken ist hierbei unter anderem an die Unterscheidung von öffentlichem Recht und Zivilrecht, an die Abgrenzung von verwaltungsrechtlichen und verfassungsrechtlichen Streitigkeiten im Rahmen des $\$ 40$ Abs. 1 VwGO oder an das Problem des Streitgegenstandes im Verwaltungsprozess, das sich wiederum auf die Auswahl der Klagearten und die Behandlung der Klageänderung auswirkt. ${ }^{130}$ Es geht bei diesen (und anderen) Themen nicht allein darum zu analysieren, wie das materielle Recht und dessen Verwirklichung im Prozess miteinander verknüpft sind (dass dies in einer vielfältigen Weise der Fall ist, steht außer Frage und ließe sich in zahlreichen Konstellationen belegen), sondern es geht um eine andere, hintergründigere Dimension dieser Zusammengehörigkeit von materiellem Recht und Prozessrecht: es geht darum, dass ein juristisches Denken in seinem Kern per definitionem ein Stück weit als ein aktionenrechtliches Denken erscheint. Dies soll freilich nicht heißen, dass man es im deutschen Verwaltungsrecht mit einer Einheit von Anspruch und Klage zu tun hat, wie sie uns vom römischen Recht her bekannt ist ${ }^{131}$ und wie sie sich im britischen Rechtskreis bis in die Gegenwart nachweisen lässt ${ }^{132}$ - dass dies nicht der Fall ist, zeigt schon ein kurzer Blick ins Gesetz. Es soll jedoch heißen, dass auch die dogmatische Behandlung materiell-rechtlicher Fragen von dieser juristischen Ur-Situation des Konflikts und des Prozesses ausgehen muss, wenn sie sich im Hinblick auf die (rechtsstaatlich geforderte) Verrechtlichung der Behördenarbeit den Grundbausteinen des juristischen Denkens zu widmen hat. Um das Gemeinte an einem Beispiel zu illustrieren, ließen sich wiederum die hergebrachten Streitfragen zu $\mathbb{4} 40$ Abs. 1 VwGO nennen: indem sie ihre Überlegungen auf den Rechtskonflikt fokussiert, sollte die dogmatische Arbeit den Grundbegriff ,Anspruch` (den Anspruch also, der mit einer Klage geltend gemacht worden ist) in das Zentrum ihrer Bemühungen um die

128 Vgl. dazu auch das Plädoyer für „Vereinfachung, Einschmelzung des Heterogenen und Zurückführung des Verworrenen auf das Wesentliche“ bei Stolleis, Entwicklungslinien (Fn. 107), S. 156.

$129 \mathrm{Zu}$ ersten Versuchen, eine verwaltungsrechtliche Dogmatik auf diesem Fundament zu errichten, siehe Haack (Fn. 94), $\$ 40$ Rnrn. 50 und 117 f.; $\$ 90$ Rn. 13; $\$ 91$ Rn. 6.

130 Es würde auf diese Weise die Dogmatik zu den einzelnen sachlichen Fragen an ein theoretisch fundiertes Gesamtkonzept rückgekoppelt - nur dies kann die Gefahr einer „Trennung von theoretisch nicht reflektiertem Handwerk und dogmatisch nicht geerdeter Theorie" (so Möllers, Verwaltungsrechtswissenschaft [Fn. 62], S. 30) reduzieren.

131 H. Honsell, Römisches Recht, 7. Aufl., Berlin 2010, S. 83.

132 Siehe dazu C. Graf von Bernstorff, Einführung in das englische Recht, 3. Aufl., München 2006, S. 4 f.; zu den historischen Hintergründen auch P. Hay, US-Amerikanisches Recht, 5. Aufl., München 2011, Rnrn. 4 ff. 
Abgrenzungen (zwischen öffentlichem Recht und Zivilrecht, aber auch zwischen verfassungsrechtlichen und sonstigen öffentlich-rechtlichen Rechtsstreitigkeiten) erheben. ${ }^{133}$ Seine Leistungskraft und seinen Wert beweist ein solches Denken vom Konflikt her insbesondere dann, wenn sich die Verrechtlichung eines Bereiches in der Anfangsphase befindet: ${ }^{134}$ erkennen ließ sich dies bei der Entwicklung eines Allgemeinen Verwaltungsrechts durch die Verwaltungsgerichte im Dialog mit der Rechtswissenschaft. ${ }^{135}$ Es stellt auch weiterhin eine reizvolle Aufgabe für Doktoranden und für andere Vertreter der Rechtswissenschaft dar, im einzelnen zu analysieren, wie sich genau dieser Vorgang derzeit vor unseren Augen vollzieht (obgleich auch hierzu schon vieles gesagt worden ist $)^{136}$ - gemeint ist damit die Herausbildung eines europäischen Verwaltungsrechts durch die Entscheidung konkreter Rechtsstreitigkeiten seitens des EuGH und des EuG. Lassen sich deutlichere Belege für einen Zusammenhang denken, den man als die „Geburt der Dogmatik aus dem Geist des Prozesses“ kennzeichnen könnte? Kurzum: allein eine solche Konzentration auf die Grundkategorien des juristischen Denkens, die unmittelbar mit dem Wesen des richterlichen Entscheidens verbunden erscheinen, vermag die Dogmatik des Verwaltungsrechts zu fundieren; hierauf zu rekurrieren, entspricht dem Anspruch verwaltungsrechtlicher Normen, in vollem Sinne Rechtsnorm zu sein. Die sogenannte ,juristische Methode“ in der Verwaltungsrechtswissenschaft könnte daher noch um vieles juristischer werden, wenn sie diese Zusammenhänge stärker als bislang beherzigt und die Situation der Klage und des Konflikts zum Dreh- und Angelpunkt ihrer Erwägungen macht. ${ }^{137}$

\section{E. Schlussbemerkungen: Gewissheiten in der Rechtswissenschaft durch die Konzentration auf den Prozess als den Kern des juristischen Denkens}

Vieles spricht dafür, dass in einer Zeit, in der das künftige Erscheinungsbild von Staatlichkeit und öffentlich-rechtlicher Ordnung ungewiss scheint, die hier dargelegte Reduktion des Programms der Wissenschaft vom Verwaltungsrecht angezeigt ist: Gewissheiten lassen sich in einer solchen Phase der Unübersichtlichkeit und der Dynamik nur dadurch gewinnen, dass man sich als Wissenschaftler auf die wesentlichen Eigenschaften des Stoffs - und dies wiederum heißt: auf das eigene Kerngeschäft - konzentriert. Nur dadurch, dass die Theorie des Verwaltungsrechts so weit wie möglich auf rechtsdogmatische Grundstrukturen zurückgeführt wird, kann man

133 Näher dazu Haack (Fn. 94), $\$ 40$ Rnrn. 50 ff.

134 Hiermit mag es zusammenhängen, dass man im juristischen Aktionendenken ein Kennzeichen „ursprünglicher“ (im Gegensatz zu „fortgeschrittenen“) Rechtskulturen erblickt hat (so E. Bucher, Für mehr Aktionendenken, AcP 186 [1986], S. 1 [7]).

135 Dazu Waldhoff, Rechtsdogmatik (Fn. 27), S. 33; allgemein dazu auch Maurer, Verwaltungsrecht (Fn. 80), $\$ 4$ Rnrn. 36 ff. (m.w.N.).

136 Siehe statt vieler T. von Danwitz, Europäisches Verwaltungsrecht, Berlin 2008, S. $166 \mathrm{ff}$. (210 ff.).

137 Ein entsprechendes Plädoyer für das zivilrechtliche Denken findet sich bei Bucher, Aktionendenken (Fn. 134), S. 72. 
das weitere Abgleiten der verwaltungsrechtlichen Lehren und folglich auch der praktischen Tätigkeit der Justiz in die Beliebigkeit stoppen, das ihnen droht, wenn sie sich von Anfang an nur darauf richten, öffentliche und private Interessen in möglichst geschmeidiger Weise abzuwägen und auszutarieren. Angeraten wird hiermit, das verwaltungsrechtliche Denken auf Begriffe zu fokussieren, die aus dem Bezug auf juristische Konfliktsituationen ihre Stichhaltigkeit und ihre Berechtigung schöpfen; auf der Grundlage solcher Begriffe ist die Dogmatik des Verwaltungsrechts zu reformieren. Die volle Rechtfertigung dafür, die Wissenschaft vom Recht der Verwaltung in dieser Form zu betreiben und eine theoretisch fundierte Art der Dogmatik selbstbewusst zu präsentieren, liegt in der Rechtsqualität des Verwaltungsrechts selbst, mit der ein juristisches Denken vom Prozess her untrennbar verknüpft ist. 